\title{
Analysis of the Impact of Ina-CBG's Rates and the Feedback Time of the Social Health Security Organizing Agency on Financial Statements Ummi Hospital Bogor
}

\author{
Muhammad Zafrullah ${ }^{1 *}$, Dr. Hadri Mulya, SE, M.Si ${ }^{1}$
}

${ }^{1}$ Master Program in Accounting at Mercubuana University Jakarta Indonesia

DOI: $10.36347 /$ sjebm.2021.v08i04.002

| Received: 16.02.2021 | Accepted: 13.04.2021 | Published: 18.04.2021

*Corresponding author: Muhammad Zafrullah

Abstract

Original Research Article

The purpose of this study was to determine the method of recording transactions in making financial reports of Ummi Bogor hospital. This study also explains whether the income and expense recognition method is in accordance with the applicable Financial Accounting Standards and the principle of matching costs against revenue. This type of research is a qualitative descriptive method using information from financial reports, financial documents and journals. The results of this study indicate that the financial statements of the Bogor Ummi hospital have basically met the Financial Accounting Standards, but there is a correction burden on the income of the Healthcare Administration Agency / Badan Penyelenggara Jaminan Sosial (National Health Insurance/NHI) patients with different periods of income recognition, which is due to the impact of using two different rates Ummi hospital regular rates and Ina-CBG's (Indonesian Case based Group's) National Health Insurance rates, then there is a feedback period for National Health Insurance patient service claims to National Health Insurance which causes medical service expenses to be recorded on a Cash basis when the payment is realized. With these implications, such hospitals must pay attention to the principle of Matching Cost against Revenue so as not to result in correntions to their income.

Keywords: Recognition Method, Income Recognition Method, Expense Recognition Method.

Copyright $(\odot) 2021$ The Author(s): This is an open-access article distributed under the terms of the Creative Commons Attribution 4.0 International License (CC BY-NC 4.0) which permits unrestricted use, distribution, and reproduction in any medium for non-commercial use provided the original author and source are credited.

\section{INTRODUCTION}

\subsection{Research Background}

Hospital is an institution engaged in the service sector. Hospitals as health service providers have the obligation to provide quality services and have accountable cost control management. This includes determining the tariff system which has been made in such a way as to the financial analysis of the local hospital and the conditions of the people in the area. In order to achieve the initial ideals of the formation of the System State Social Security (SJSN) and Social Security. Administering Bodies (National Health Insurance) For this Health, a proper tariff determination system is needed guaranteeing that neither party is harmed, both patients (participants SJSN), hospital or other designated health agency (provider services), as well as National Health Insurance. For this reason, proper service systems and procedures need to be carefully planned. Although there is no clear tariff determination yet, the latest discourse points to a system that refers to Indonesian Case Based Groups (INA-CBG's). INA-CBG's itself is a payment system to health care providers grouped based on the same clinical characteristics and use of resources (the same cost of care). Unlike the payment system based on the number of services or package payment systems grouped based on similar services. Service patterns or package systems that are grouped based on similar services, the payment pattern with this system is a prospective payment where the fee is determined before the service is provided. Currently, payments are made based on the type of service or service package. In the INA-CBG's pattern, there is a per-case episode rate determined by the INA-CBG's code. Payment per-code INA-CBG's includes costs from the time the patient enters the hospital until the patient goes home or recovers according to a predetermined clinical pathway.

One tariff is paid at once for all service components which include doctor's examinations, diagnostic support (laboratory, radio diagnostic, and electromedical), medicines, as well as inpatient class accommodation for inpatients. The problem that arises at the Bogor City UMMI Hospital as the object of research is that because there are two rates used, a correction will appear in the hospital records in the 
account "Loss or gain on National Health Insurance difference" and feedback from National Health Insurance takes 2 months to 6 months, clearing bills to National Health Insurance for refusals, some of which are up to one year or even more than one year, so that the adjustment of the National Health Insurance difference is recorded on a cash basis, while the income with Accrual Basis treatment, as well as calculations of doctor and other medical services are also recorded on a cash basis the meaning of recording income and expenses is not in the same period.

This form of recording violates statement of financial accounting standards Matching costs against revenue, where the revenue and expenses that follow are not recorded in the same period and cause the information presented in the financial statements to be inaccurate for users of the financial statements. Based on the background of the problems above, it is necessary to make a formulation the problem is "What is the impact of the National Health Insurance using the INA-CBG's rate system and the feedback period of claims for health services on financial records and reports at the UMMI Hospital, Bogor City?"

\section{LITERATURE REVIEWS}

\subsection{Definition and Purpose of Financial Statements}

The existence of an obligation for a business entity to prepare financial statements making these financial statements an integral part of the business entity itself. Financial reports are accounting products. The term committee of the American Institute of Certified Public Accountants (AICPA) defines accounting as follows [1], an information system that measures business activities to process this information into reports, and communicate the results to accounting decision makers, often referred to as business language. This language can be viewed as an information system that provides essential information about the financial activities of an entity to various individuals or groups to be used in making informed judgments and decisions. The financial report according to Kieso [2] is to provide; information that is useful in making investment and credit decisions, information that is useful in assessing the prospects for future cash flows, and information about the resources the company claims against these resources, and changes therein.

The preparation of the financial statements itself is based on several basic accounting principles, one of which is the Matching Cost Against Revenue Principle which in this principle requires the use of an accrual basis in such a way that the recognized income is matched with the related expenses incurred in generating revenue. According to Beechy [3], the cash basis recognizes or records transactions or events only when cash is received or when cash is issued, while liabilities are not presented in the financial statements as well as receivables and other assets. According to Mulyadi [4] the accrual-based accounting system is an accounting basis in which economic transactions or accounting events are recognized, recorded and presented in financial statements based on the effects of transactions at the time the transactions occur, regardless of when cash is received or paid.

\subsection{Definition of Income}

The definition of income issued by the Indonesian Institute of Accountants [5]: Income is the gross inflow of economic benefits arising from the normal activities of the company during a period when the inflows result in an increase in equity that does not come from investment contributions. According to Kieso [2]: Revenue is an inflow of assets or other increase in the entity's assets or the payment of its liabilities (or a combination of the two) during a period, which is caused by the delivery or production of goods, provision of services, or other activities that are part of the main operation. or corporate central operations. The income earned by the company must be classified so that the source of the income can be identified. By knowing the source of income, the company can evaluate the target to be obtained. Soemarso [4] also explains, Revenue is the amount charged to subscriptions for goods and services sold. Income is also defined as the gross increase in capital (usually through the receipt of an asset from a subscription) arising from the goods and services sold. Income reporting requires not only a statement that the firm has produced economic value in the form of goods or services, but also a measure of value itself. Income should be identified with the period during which the primary economic activity necessary to create and distribute goods and services has been completed, provided that objective measures of the results of those activities are available.

\subsection{Definition of Expenses}

Cost is cash or cash equivalent value sacrificed for goods and services that are expected to provide benefits at present or in the future. While expenses are expenses that have been used or used (expired cost). Expenses in a broad sense include all expired expenses that are deducted from revenue.

\subsection{The Principle of Considering Income and Expenses}

Revenues earned and expenses incurred in a given accounting period should be properly matched to allow reasonable operating results. In order for income and expenses to be properly matched, it is necessary to first know the purpose of the principle of matching costs against the revenue principle. The presentation of income and expenses in the financial statements cannot be separated from the accounting method used, because financial statements are the result of the accounting process established by the company. The methods that can be used are as follows [2]: 1. Cash Basis Accounting Methods. 2. Accrual Basis Accounting Methods (Accrual Basis Accounting). 


\section{RESEARCH DESIGN AND METHODS}

\subsection{Research Scope}

The scope of research studied in this study is about recording using two rates in the accrual basis and cash basis methods in recording the bills of the Bogor City UMMI Hospital to National Health Insurance from 2016 to the end of 2018 regarding income and expense accounts in the report. UMMI Hospital's financial report for the period ended December 31, 2016, 2017 and 2018, as well as a feedback period for hospital health service claims to National Health Insurance which affected the recording treatment of the resulting burdens. The objects in this study are the financial statements of the Bogor UMMI Hospital for 2016 to 2018, general ledger, details of income and expenses, details of accounts receivable, proof of payment requests, proof of bank and cash expenditures and other accounts related to research.

\subsection{Types and Sources of Data}

The data used in this study are primary data and secondary data. Primary data is data that is obtained directly from the object under study, which requires further processing. The data is in the form of field research results in the finance section related to the recognition of income and expenses at UMMI Hospital Bogor, including master hospital rates and coding results with INA-CBG's National Health Insurance rates. Secondary data is data obtained from companies that have been processed and documented in the company. Secondary data can generally be in the form of evidence, notes, or historical reports, magazines, articles that have been compiled in archives both published and unpublished. In this case, secondary data is taken in the form of UMMI hospital financial reports from 2016 to 2018, general ledger and income details based on master hospital rates and INA-CBG's National Health Insurance rates for the period 2016 to 2018. The data analysis in this research is descriptive qualitative analysis, which is an analysis that describes a situation objectively to draw conclusions about the observed data.

\section{RESULTS AND DISCUSSION \\ 4.1 Presentation of Financial Statements for UMMI Hospital Bogor}

At the beginning of its establishment in 2012, the UMMI Hospital with its legality PT Dutagraha Afiah in Bogor obtained a license to run a business in the field of hospitals with special services for mothers and children so that the name was UMMI Mother and Child Hospital. Then in 2016 it changed to become a public hospital that serves general patients so that the name changed to UMMI Hospital. The following is the UMMI Hospital Profit Loss Report for 2016, 2017 and 2018:

\begin{tabular}{|c|c|c|c|c|}
\hline \multicolumn{5}{|l|}{$\begin{array}{l}\text { RS UMMI } \\
\text { Income Statement } \\
2016\end{array}$} \\
\hline Outpatient Income & $707,578,050$ & $635,962,200$ & $782,109,968$ & $836,338,161$ \\
\hline Inpatient Income & $2,052,256,408$ & $1,793,955,938$ & $2,439,785,024$ & 2,392,845,193 \\
\hline Medical and Pharmaceutical Support Income & $1,237,036,018$ & $1,398,640,543$ & $1,630,023,876$ & $1,549,868,850$ \\
\hline The Ammount of Gross Operating Income & $3,996,870,476$ & $3,828,558,681$ & $4,851,918,868$ & $4,779,052,204$ \\
\hline Difference in National Health Insurance & $(340,892,745)$ & $(277,631,674)$ & $(384,268,146)$ & $(564,560,897)$ \\
\hline The Ammount of Net Operating Income & $3,655,977,731$ & $3,550,927,007 "$ & $4,467,650,722$ & $4,214,491,307$ \\
\hline \multicolumn{5}{|l|}{ Direct Operating Expenses } \\
\hline Doctor's Fees & $931,736,697$ & $982,390,125$ & $1,075,788,445$ & $614,148,409$ \\
\hline Medical Support Burden & $20,398,558$ & $11,998,484$ & $27,352,983$ & $31,838,469$ \\
\hline Medical Operating Expenses & $190,460,899$ & $212,831,557$ & $235,402,821$ & $281,182,182$ \\
\hline Pharmaceutical Expenses & $633,201,051$ & $636,975,778$ & $792,256,483$ & $714,248,371$ \\
\hline Other Direct Expenses & $86,961,929$ & $44,175,472$ & $45,945,709$ & $36,936,182$ \\
\hline Total Direct Operating Expenses & $1,862,759,134$ & $1,888,371,416$ & $2,176,746,441$ & $1,678,353,613$ \\
\hline \multicolumn{5}{|l|}{ Indirect Operating Expenses } \\
\hline Staffing Burden & $920,946,385$ & $928,531,088$ & $1,207,457,168$ & $964,779,106$ \\
\hline Ajustments for The Difference in Stock & $1,408,916$ & $22,534,128$ & $15,189,725$ & $(4,018,065)$ \\
\hline Total Indirect Operating Expenses & $922,355,301$ & $951,065,216$ & $1,222,646,893$ & $960,761,041$ \\
\hline Total Operating Expenses & $2,785,114,435$ & $2,839,436,632$ & $3,399,393,334$ & $2,639,114,654$ \\
\hline Gross Profit & $870,863,296$ & $711,490,375$ & $1,068,257,388$ & $1,575,376,653$ \\
\hline \multicolumn{5}{|l|}{ Operating Expenses } \\
\hline General and Administrative Expenses & $130,421,740$ & $121,686,025$ & $200,073,682$ & $257,284,461$ \\
\hline Utility Expense & $85,539,493$ & $85,860,710$ & $83,341,805$ & $96,685,206$ \\
\hline Marketing Operational Expenses & $16,756,650$ & $10,014,500$ & $11,167,229$ & $1,558,125$ \\
\hline Maintenance and Repair Expenses & $18,333,625$ & $23,435,070$ & $21,256,760$ & $34,419,920$ \\
\hline Depreciation Expense & $364,472,081$ & $365,781,337$ & $365,585,920$ & $367,423,180$ \\
\hline Total Operating Expenses & $615,523,589$ & $606,777,642$ & $681,425,396$ & $757,370,892$ \\
\hline \multicolumn{5}{|l|}{ Other Income / Expenses } \\
\hline Non Operating Income & $108,826,163$ & $12,379,052$ & $87,325,418$ & $16,316,668$ \\
\hline Non Operating Expenses & $436,021,756$ & $67,121,614$ & $787,862,616$ & $425,004,464$ \\
\hline The Ammount of Other Income/Expenses & $(327,195,593)$ & $(54,742,562)$ & $(700,537,198)$ & $(408,687,796)$ \\
\hline Net Profit & $(\mathbf{7 1 , 8 5 5 , 8 8 6 )}$ & $49,970,171$ & $(313,705,206)$ & $409,317,965$ \\
\hline
\end{tabular}




\begin{tabular}{|c|c|c|c|c|}
\hline \multicolumn{5}{|l|}{$\begin{array}{l}\text { RS UMMI } \\
\text { Income Statement } \\
2016\end{array}$} \\
\hline Outpatient Income & $936,275,200$ & $855,663,350$ & $747,521,800$ & $1,003,207,000$ \\
\hline Inpatient Income & $2,344,732,356$ & $2,072,679,659$ & $2,075,157,285$ & $2,299,055,704$ \\
\hline Medical and Pharmaceutical Support Income & $1,758,423,045$ & $1,661,828,712$ & $1,592,633,989$ & $1,812,856,582$ \\
\hline The Ammount of Gross Operating Income & $5,039,430,601$ & $4,590,171,721$ & $4,415,313,074$ & $5,115,119,286$ \\
\hline Difference in National Health Insurance & $(\mathbf{7 8 8 , 7 9 5 , 7 4 8 )}$ & $(832,886,055)$ & $(769,103,673)$ & $(816,285,433)$ \\
\hline The Ammount of Net Operating Income & $4,250,634,853$ & $3,757,285,666$ & $3,646,209,401 "$ & $4,298,833,853$ \\
\hline \multicolumn{5}{|l|}{ Direct Operating Expenses } \\
\hline Doctor's Fees & $1,057,771,951$ & $1,068,417,251$ & $993,500,786$ & $640,253,372$ \\
\hline Medical Support Burden & 29,985,152 & $28,052,092$ & $21,555,264$ & $35,234,670$ \\
\hline Medical Operating Expenses & $212,026,178$ & $306,706,898$ & $233,972,823$ & $257,685,837$ \\
\hline Pharmaceutical Expenses & $814,982,298$ & $666,265,241$ & $589,087,310$ & $635,337,570$ \\
\hline Other Direct Expenses & $47,762,242$ & $56,418,726$ & $35,537,561$ & $50,412,199$ \\
\hline Total Direct Operating Expenses & $2,162,527,821$ & $2,125,860,208$ & $1,873,653,744$ & $1,618,923,648$ \\
\hline \multicolumn{5}{|l|}{ Indirect Operating Expenses } \\
\hline Staffing Burden & $1,052,096,738$ & $1,503,691,917$ & $1,175,069,384$ & $1,266,772,377$ \\
\hline Ajustments for The Difference in Stock & $(\mathbf{2 3 5}, \mathbf{8 9 5 , 5 1 0 )}$ & $\mathbf{9 0 , 8 3 9 , 8 7 6}$ & $67,986,855$ & $10,307,300$ \\
\hline Total Indirect Operating Expenses & $816,201,228$ & $1,594,531,793$ & $1,243,056,239$ & $1,277,079,677$ \\
\hline Total Operating Expenses & $2,978,729,049$ & $3,720,392,001$ & $3,116,709,983$ & $2,896,003,325$ \\
\hline Gross Profit & 1,271,905,804 & $36,893,665$ & $529,499,418$ & $1,402,830,528$ \\
\hline \multicolumn{5}{|l|}{ Operating Expenses } \\
\hline General and Administrative Expenses & $325,804,028$ & $257,605,138$ & $145,437,153$ & $184,910,852$ \\
\hline Utility Expense & $95,797,295$ & $97,925,052$ & $96,666,930$ & $92,433,491$ \\
\hline Marketing Operational Expenses & $6,293,315$ & $36,470,675$ & $4,667,120$ & $13,792,494$ \\
\hline Maintenance and Repair Expenses & $24,963,430$ & $18,814,480$ & $35,687,340$ & $47,721,934$ \\
\hline Depreciation Expense & $368,261,680$ & $375,429,934$ & $376,336,658$ & $377,746,053$ \\
\hline Total Operating Expenses & $821,119,748$ & $786,245,279$ & $658,795,201$ & $716,604,824$ \\
\hline \multicolumn{5}{|l|}{ Other Income / Expenses } \\
\hline Non Operating Income & $118,589,494$ & $26,730,743$ & $10,724,650$ & $134,167,653$ \\
\hline Non Operating Expenses & $416,815,864$ & $4,603,674$ & $716,767,275$ & $358,917,323$ \\
\hline The Ammount of Other Income/Expenses & $(298,226,370)$ & $22,127,069$ & $(706,042,625)$ & $(224,749,670)$ \\
\hline Net Profit & $152,559,686$ & $(\mathbf{7 2 7 , 2 2 4 , 5 4 5 )}$ & $(835,338,408)$ & $461,476,034$ \\
\hline
\end{tabular}




\begin{tabular}{|c|c|c|c|c|c|}
\hline \multicolumn{6}{|l|}{$\begin{array}{l}\text { RS UMMI } \\
\text { Income Statement } \\
2016\end{array}$} \\
\hline Outpatient Income & $942,833,800$ & $963,945,000$ & $995,545,200$ & $972,681,203$ & $10,379,660,932$ \\
\hline Inpatient Income & 2,373,927,502 & 2,369,019,589 & $2,203,154,058$ & $2,174,217,937$ & $26,590,786,653$ \\
\hline Medical and Pharmaceutical Support Income & $1,856,807,413$ & $1,991,293,411$ & $1,880,529,592$ & $1,920,761,262$ & $20,290,703,293$ \\
\hline The Ammount of Gross Operating Income & $5,173,568,715$ & $5,324,258,000$ & $5,079,228,850$ & $5,067,660,402$ & $57,261,150,878$ \\
\hline Difference in National Health Insurance & $(772,873,073)$ & $(685,595,924)$ & $(542,560,435)$ & $(184,943,397)$ & $(6,960,397,200)$ \\
\hline The Ammount of Net Operating Income & $4,400,695,6422^{\top}$ & $4,638,662,076$ & $4,536,668,415$ & $4,882,717,005$ & $50,300,753,678$ \\
\hline \multicolumn{6}{|l|}{ Direct Operating Expenses } \\
\hline Doctor's Fees & $1,483,756,741$ & $1,359,659,378$ & $641,176,065$ & $1,078,067,867$ & $11,926,667,087$ \\
\hline Medical Support Burden & $32,940,646$ & $37,525,409$ & $32,659,542$ & 29,867,604 & $339,408,873$ \\
\hline Medical Operating Expenses & 289,791,941 & $297,289,906$ & $291,227,314$ & $199,726,218$ & $3,008,304,574$ \\
\hline Pharmaceutical Expenses & $647,114,480$ & $661,447,430$ & $623,197,372$ & $657,764,262$ & $8,071,877,646$ \\
\hline Other Direct Expenses & $49,271,465$ & $44,964,956$ & $44,327,175$ & $50,248,380$ & $592,961,996$ \\
\hline Total Direct Operating Expenses & $2,502,875,273$ & 2,400,887,079 & $1,632,587,468$ & $2,015,674,331$ & $23,939,220,176$ \\
\hline \multicolumn{6}{|l|}{ Indirect Operating Expenses } \\
\hline Staffing Burden & $1,253,341,205$ & $1,299,629,349$ & $1,279,713,033$ & $1,270,046,800$ & $14,122,074,550$ \\
\hline Ajustments for The Difference in Stock & $\mathbf{1 7 1 , 8 3 3 , 5 5 1}$ & $83,079,634$ & $41,089,525$ & $(21,575,040)$ & $242,780,895$ \\
\hline Total Indirect Operating Expenses & $1,425,174,756$ & $1,382,708,983$ & $1,320,802,558$ & $1,248,471,760{ }^{\circ}$ & $14,364,855,445$ \\
\hline Total Operating Expenses & $3,928,050,029$ & $3,783,596,062$ & $2,953,390,026$ & $3,264,146,0911^{\prime}$ & $38,304,075,621$ \\
\hline Gross Profit & $472,645,613$ & $855,066,014$ & $1,583,278,389$ & $1,618,570,914$ & $11,996,678,057$ \\
\hline \multicolumn{6}{|l|}{ Operating Expenses } \\
\hline General and Administrative Expenses & $163,451,051$ & $141,803,125$ & $115,546,362$ & $651,556,126$ & $2,695,579,743$ \\
\hline Utility Expense & $82,614,235$ & $82,522,385$ & $85,689,586$ & $85,778,253$ & $1,070,854,441$ \\
\hline Marketing Operational Expenses & $10,902,657$ & $17,548,831$ & $17,618,278$ & $17,644,700$ & $164,434,574$ \\
\hline Maintenance and Repair Expenses & $16,976,540$ & $29,989,840$ & $27,902,290$ & $14,215,160$ & $313,716,389$ \\
\hline Depreciation Expense & $408,865,251$ & $409,643,635$ & $408,944,174$ & $411,024,127$ & $4,599,514,031$ \\
\hline Total Operating Expenses & $682,809,734$ & $681,507,816$ & $655,700,690$ & $1,180,218,366$ & $8,844,099,178$ \\
\hline \multicolumn{6}{|l|}{ Other Income / Expenses } \\
\hline Non Operating Income & $23,734,948$ & $26,460,382$ & $23,099,596$ & $19,133,716$ & $607,488,483$ \\
\hline Non Operating Expenses & $616,649,036$ & $159,409,753$ & $481,446,523$ & $107,577,573$ & $4,578,197,471$ \\
\hline The Ammount of Other Income/Expenses & $(592,914,088)$ & $(132,949,371)$ & $(458,346,927)$ & $(88,443,857)$ & $(3,970,708,988)$ \\
\hline Net Profit & $(803,078,209)$ & $40,608,827$ & $469,230,772$ & $349,908,690$ & $(818,130,109)$ \\
\hline
\end{tabular}




\begin{tabular}{|c|c|c|c|c|}
\hline \multicolumn{5}{|l|}{$\begin{array}{l}\text { RS UMMI } \\
\text { Income Statement } \\
2017\end{array}$} \\
\hline Outpatient Income & $1,032,545,390$ & $1,058,405,100$ & $1,430,427,500$ & $1,288,602,650$ \\
\hline Inpatient Income & $2,452,887,972$ & $2,615,470,282$ & 2,881,905,738 & $2,888,543,524$ \\
\hline Medical and Pharmaceutical Support Income & $2,083,020,434$ & $1,966,160,790$ & $2,132,312,670$ & $1,950,557,986$ \\
\hline $\begin{array}{l}\text { The Ammount of Gross Operating Income } \\
\text { Difference in National Health Insurance }\end{array}$ & $\begin{array}{r}5,568,453,796 \\
(995,280,119)\end{array}$ & $\begin{array}{r}5,640,036,172 \\
(623,589,422)\end{array}$ & $\begin{array}{c}6,444,645,908 \\
(655,891,348)\end{array}$ & $\begin{array}{r}6,127,704,160 \\
(1,069,592,651)\end{array}$ \\
\hline The Ammount of Net Operating Income & $4,573,173,677$ & $5,016,446,750$ & $5,788,754,560$ & $5,058,111,509$ \\
\hline \multicolumn{5}{|l|}{ Direct Operating Expenses } \\
\hline Doctor's Fees & $1,474,207,072$ & $1,019,963,760$ & $1,024,499,851$ & $729,295,981$ \\
\hline Medical Support Burden & $148,060,703$ & $\mathbf{7 1 , 4 8 6 , 4 8 1}$ & $98,307,965$ & $109,884,339$ \\
\hline Medical Operating Expenses & $256,021,527$ & $81,716,935$ & $255,591,809$ & $204,002,844$ \\
\hline Pharmaceutical Expenses & $735,621,135$ & $759,242,160$ & $719,438,680$ & $668,001,089$ \\
\hline Other Direct Expenses & $53,884,245$ & $51,293,977$ & $51,822,218$ & $47,300,363$ \\
\hline Total Direct Operating Expenses & $2,667,794,682$ & $1,983,703,313$ & $2,149,660,523$ & $1,758,484,616$ \\
\hline \multicolumn{5}{|l|}{ Indirect Operating Expenses } \\
\hline Staffing Burden & $1,383,491,090$ & $1,285,083,350$ & $1,333,795,674$ & $1,322,906,077$ \\
\hline Ajustments for The Difference in Stock & $(7,780,842)$ & $3,109,544$ & $5,402,952$ & $(10,093,789)$ \\
\hline Total Indirect Operating Expenses & $1,375,710,248$ & $1,288,192,894$ & $1,339,198,626$ & $1,312,812,288$ \\
\hline Total Operating Expenses & $4,043,504,930$ & $3,271,896,207$ & $3,488,859,149$ & $3,071,296,904$ \\
\hline Gross Profit & $529,668,747$ & $1,744,550,543$ & $2,299,895,411$ & 1,986,814,605 \\
\hline \multicolumn{5}{|l|}{ Operating Expenses } \\
\hline General and Administrative Expenses & $98,362,319$ & $244,160,722$ & $242,619,528$ & $70,406,633$ \\
\hline Utility Expense & $88,023,473$ & $73,642,955$ & $78,078,380$ & $76,966,148$ \\
\hline Marketing Operational Expenses & $\mathbf{8 9 1 , 1 8 0}$ & $1,660,000$ & $1,451,445$ & $1,919,498$ \\
\hline Maintenance and Repair Expenses & $21,801,130$ & $29,963,482$ & $57,990,972$ & $17,263,570$ \\
\hline Depreciation Expense & $379,231,096$ & $380,579,579$ & $364,770,171$ & $348,169,320$ \\
\hline Total Operating Expenses & $588,309,198$ & $730,006,738$ & $744,910,496$ & $514,725,169$ \\
\hline \multicolumn{5}{|l|}{ Other Income / Expenses } \\
\hline Non Operating Income & $17,796,315$ & $97,487,462$ & $78,059,021$ & $116,007,088$ \\
\hline Non Operating Expenses & $421,951,350$ & $518,156,931$ & $486,631,085$ & $432,596,297$ \\
\hline The Ammount of Other Income/Expenses & $(404,155,035)$ & $(420,669,469)$ & $(408,572,064)$ & $(316,589,209)$ \\
\hline Net Profit & $(462,795,486)$ & $593,874,336$ & $1,146,412,851$ & $1,155,500,227$ \\
\hline
\end{tabular}




\begin{tabular}{|c|c|c|c|c|}
\hline $\begin{array}{l}\text { RS UMMI } \\
\text { Income Statement } \\
2017\end{array}$ & May & Jun & Jul & Aug \\
\hline Outpatient Income & $1,574,315,050$ & $1,181,814,700$ & $1,654,110,700$ & $1,788,280,700$ \\
\hline Inpatient Income & $3,016,316,731$ & 2,567,028,897 & $3,411,698,620$ & $3,615,662,030$ \\
\hline Medical and Pharmaceutical Support Income & $2,049,635,108$ & $1,750,053,158$ & $2,184,294,127$ & $2,393,930,030$ \\
\hline The Ammount of Gross Operating Income & $6,640,266,889$ & $5,498,896,755$ & $7,250,103,447$ & $7,797,872,760$ \\
\hline Difference in National Health Insurance & $(1,352,150,497)$ & $(1,138,093,655)$ & $(1,125,871,057)$ & $(1,416,461,403)$ \\
\hline The Ammount of Net Operating Income & $5,288,116,392$ & $4,360,803,100$ & $6,124,232,390$ & $6,381,411,357$ \\
\hline Direct Operating Expenses & & & & \\
\hline Doctor's Fees & $1,423,754,056$ & $1,138,270,005$ & $1,222,588,363$ & $1,198,289,477$ \\
\hline Medical Support Burden & $129,289,921$ & $99,806,265$ & $132,922,813$ & $98,265,524$ \\
\hline Medical Operating Expenses & $205,540,934$ & $170,831,312$ & $209,449,969$ & 219,114,688 \\
\hline Pharmaceutical Expenses & $681,696,377$ & $585,218,717$ & $700,449,324$ & $747,337,729$ \\
\hline Other Direct Expenses & $6,696,520$ & $22,798,322$ & $17,169,584$ & $2,989,407$ \\
\hline Total Direct Operating Expenses & $2,446,977,808$ & $2,016,924,621$ & $2,282,580,053$ & $2,265,996,825$ \\
\hline Indirect Operating Expenses & & & & \\
\hline Staffing Burden & $1,331,040,525$ & $2,310,556,696$ & $1,358,063,437$ & $1,275,557,257$ \\
\hline Ajustments for The Difference in Stock & $(1,974,940)$ & $1,133,687$ & $(534,928)$ & $(373,254)$ \\
\hline Total Indirect Operating Expenses & $1,329,065,585$ & $2,311,690,383$ & 1,357,528,509 & $1,275,184,003$ \\
\hline Total Operating Expenses & $3,776,043,393$ & $4,328,615,004$ & $3,640,108,562$ & $3,541,180,828$ \\
\hline Gross Profit & $1,512,072,999$ & $32,188,096$ & $2,484,123,828$ & $2,840,230,529$ \\
\hline Operating Expenses & & & & \\
\hline General and Administrative Expenses & $138,933,559$ & $109,137,226$ & $409,655,323$ & $130,944,654$ \\
\hline Utility Expense & $80,804,542$ & $85,658,065$ & $\mathbf{7 3 , 5 3 8 , 9 7 1}$ & $83,602,460$ \\
\hline Marketing Operational Expenses & $1,174,145$ & 497,441 & $1,566,142$ & 2,386,240 \\
\hline Maintenance and Repair Expenses & $77,469,636$ & $15,523,617$ & $33,397,697$ & $106,116,355$ \\
\hline Depreciation Expense & $358,091,089$ & $363,591,158$ & $348,233,137$ & $358,417,267$ \\
\hline Total Operating Expenses & $656,472,971$ & $574,407,507$ & $866,391,270$ & $681,466,976$ \\
\hline Other Income / Expenses & & & & \\
\hline Non Operating Income & $67,749,102$ & $23,046,492$ & $38,298,961$ & $30,122,150$ \\
\hline Non Operating Expenses & $412,865,556$ & $469,081,730$ & $401,355,124$ & $396,909,833$ \\
\hline The Ammount of Other Income/Expenses & $(345,116,454)$ & $(446,035,238)$ & $(363,056,163)$ & $(366,787,683)$ \\
\hline Net Profit & $510,483,574$ & $(988,254,649)$ & $1,254,676,395$ & $1,791,975,870$ \\
\hline
\end{tabular}




\begin{tabular}{|c|c|c|c|c|c|}
\hline $\begin{array}{l}\text { RS UMMI } \\
\text { Income Statement } \\
2017 \quad \text { Account }\end{array}$ & Sep & Oct & Nov & Dec & Total \\
\hline Outpatient Income & $1,549,606,100$ & $1,733,104,277$ & $1,813,923,400$ & $1,845,931,900$ & $17,951,067,467$ \\
\hline Inpatient Income & $3,264,247,533$ & $3,702,903,494$ & $3,826,499,168$ & $4,199,366,702$ & $38,442,530,691$ \\
\hline Medical and Pharmaceutical Support Income & $2,378,493,985$ & $2,581,863,239$ & 2,544,623,911 & $2,625,112,818$ & $26,640,058,256$ \\
\hline The Ammount of Gross Operating Income & $7,192,347,618$ & $8,017,871,010$ & $8,185,046,479$ & $8,670,411,420$ & $83,033,656,414$ \\
\hline Difference in National Health Insurance & $(464,104,015)$ & $(2,008,015,636)$ & $(4,609,375,370)$ & $(4,093,711,301)$ & $(19,552,136,474)$ \\
\hline The Ammount of Net Operating Income & $6,728,243,603$ & $6,009,855,374$ & $3,575,671,109$ & $4,576,700,119$ & $63,481,519,940$ \\
\hline Direct Operating Expenses & & & & & \\
\hline Doctor's Fees & $818,760,463$ & $847,957,440$ & $1,179,599,237$ & $2,150,016,240$ & $14,227,201,945$ \\
\hline Medical Support Burden & $294,269,744$ & $179,985,638$ & $188,805,201$ & $399,087,558$ & $1,950,172,152$ \\
\hline Medical Operating Expenses & $155,989,824$ & $198,393,482$ & $176,166,291$ & $219,156,666$ & $2,351,976,281$ \\
\hline Pharmaceutical Expenses & $816,886,185$ & $787,112,174$ & $767,205,943$ & $1,789,739,964$ & $9,757,949,477$ \\
\hline Other Direct Expenses & $50,781,325$ & $66,025,079$ & $4,418,137$ & $198,655,158$ & $573,834,335$ \\
\hline Total Direct Operating Expenses & $2,136,687,541$ & $2,079,473,813$ & $2,316,194,809$ & $4,756,655,586$ & $28,861,134,190$ \\
\hline Indirect Operating Expenses & & & & & \\
\hline Staffing Burden & $1,404,305,049$ & $1,411,158,034$ & $1,411,510,570$ & $1,485,248,608$ & $17,312,716,367$ \\
\hline Ajustments for The Difference in Stock & $1,173,000$ & $(715,591)$ & $(1,804,956)$ & $326,665,550$ & $314,206,433$ \\
\hline Total Indirect Operating Expenses & $1,405,478,049$ & $1,410,442,443$ & $1,409,705,614$ & $1,811,914,158$ & $17,626,922,800$ \\
\hline Total Operating Expenses & $3,542,165,590$ & $3,489,916,256$ & $3,725,900,423$ & $6,568,569,744$ ' & $46,488,056,990$ \\
\hline Gross Profit & $3,186,078,013$ & 2,519,939,118 & $(150,229,314)$ & $(1,991,869,625)$ & $16,993,462,950$ \\
\hline Operating Expenses & & & & & \\
\hline General and Administrative Expenses & $140,548,123$ & $159,932,954$ & $209,526,924$ & $165,286,978$ & $2,119,514,943$ \\
\hline Utility Expense & $90,124,420$ & $86,702,201$ & $97,145,904$ & $106,918,034$ & $1,021,205,553$ \\
\hline Marketing Operational Expenses & 543,309 & $2,981,000$ & $4,846,052$ & 7,530,931 & $27,447,383$ \\
\hline Maintenance and Repair Expenses & $33,394,280$ & $54,427,837$ & $14,893,407$ & $103,591,511$ & $565,833,494$ \\
\hline Depreciation Expense & $362,535,157$ & $363,736,060$ & $1,235,205,755$ & $318,156,948$ & $5,180,716,737$ \\
\hline Total Operating Expenses & $627,145,289$ & $667,780,052$ & $1,561,618,042$ & $701,484,402$ & $8,914,718,110$ \\
\hline Other Income / Expenses & & & & & \\
\hline Non Operating Income & $84,297,235$ & $109,017,440$ & $15,794,081$ & $100,539,317$ & $778,214,664$ \\
\hline Non Operating Expenses & $400,911,677$ & $385,574,667$ & $407,701,390$ & $365,939,574$ & $5,099,675,214$ \\
\hline The Ammount of Other Income/Expenses & $(316,614,442)$ & $(276,557,227)$ & $(391,907,309)$ & $(265,400,257)$ & $(4,321,460,550)$ \\
\hline Net Profit & $2,242,318,282$ & $1,575,601,839$ & $(2,103,754,665)$ & $(2,958,754,284)$ & $3,757,284,290$ \\
\hline
\end{tabular}




\begin{tabular}{|c|c|c|c|c|}
\hline \multicolumn{5}{|l|}{$\begin{array}{l}\text { RS UMMI } \\
\text { Income Statement } \\
2018\end{array}$} \\
\hline Outpatient Income & $2,406,410,900$ & $2,095,016,000$ & $1,452,756,812$ & $1,487,492,300$ \\
\hline Inpatient Income & $5,041,172,789$ & $3,558,212,305$ & $3,543,176,113$ & $3,241,780,701$ \\
\hline Medical and Pharmaceutical Support Income & $3,069,520,380$ & $2,386,480,144$ & $2,475,101,372$ & $2,550,417,210$ \\
\hline The Ammount of Gross Operating Income & $10,517,104,069$ & $8,039,708,449$ & $7,471,034,297$ & $7,279,690,211$ \\
\hline Difference in National Health Insurance & $30,492,240$ & $(5,130,407)$ & $(4,038,896,202)$ & $(1,903,097,819)$ \\
\hline The Ammount of Net Operating Income & $10,547,596,309$ & $8,034,578,042$ & $3,432,138,095$ & $5,376,592,392$ \\
\hline \multicolumn{5}{|l|}{ Direct Operating Expenses } \\
\hline Doctor's Fees & $533,132,143$ & $442,111,791$ & $1,885,494,841$ & $1,687,567,680$ \\
\hline Medical Support Burden & $167,207,540$ & $137,806,730$ & 73,502,986 & $141,857,281$ \\
\hline Medical Operating Expenses & $341,212,924$ & $278,809,309$ & $300,068,826$ & $215,014,438$ \\
\hline Pharmaceutical Expenses & $1,054,851,772$ & $836,415,895$ & $1,121,903,614$ & $993,943,481$ \\
\hline Other Direct Expenses & $63,388,314$ & $15,960,133$ & $63,130,952$ & $15,988,669$ \\
\hline Total Direct Operating Expenses & $2,159,792,693$ & $1,711,103,858$ & $3,444,101,219$ & $3,054,371,549$ \\
\hline \multicolumn{5}{|l|}{ Indirect Operating Expenses } \\
\hline Staffing Burden & $1,638,489,408$ & $1,693,591,082$ & $\mathbf{1 , 5 9 0 , 5 8 7 , 1 5 1}$ & $1,784,637,340$ \\
\hline Ajustments for The Difference in Stock & $(6,759,144)$ & $45,627,738$ & $(118,625,223)$ & $(35,005,208)$ \\
\hline Total Indirect Operating Expenses & $1,631,730,264$ & $1,739,218,820$ & $1,471,961,928$ & $1,749,632,132$ \\
\hline Total Operating Expenses & $3,791,522,957$ & $3,450,322,678$ & $4,916,063,147$ & $4,804,003,681$ \\
\hline Gross Profit & $6,756,073,352$ & $4,584,255,364$ & $(1,483,925,052)$ & $572,588,711$ \\
\hline \multicolumn{5}{|l|}{ Operating Expenses } \\
\hline General and Administrative Expenses & $113,491,266$ & $146,167,523$ & $164,038,335$ & $216,480,122$ \\
\hline Utility Expense & $94,176,737$ & $105,549,557$ & $90,395,818$ & $113,361,619$ \\
\hline Marketing Operational Expenses & $83,189,500$ & $2,630,836$ & $10,590,359$ & $15,690,800$ \\
\hline Maintenance and Repair Expenses & $30,532,550$ & $20,264,817$ & $27,573,765$ & $50,742,120$ \\
\hline Depreciation Expense & $423,059,907$ & $405,863,667$ & $411,483,597$ & $404,859,950$ \\
\hline Total Operating Expenses & $744,449,960$ & $680,476,400$ & $704,081,873$ & $801,134,611$ \\
\hline \multicolumn{5}{|l|}{ Other Income / Expenses } \\
\hline Non Operating Income & $69,984,282$ & $17,532,772$ & $17,656,824$ & $585,138,880$ \\
\hline Non Operating Expenses & $368,689,317$ & $347,493,700$ & $343,463,007$ & $348,121,090$ \\
\hline The Ammount of Other Income/Expenses & $(298,705,035)$ & $(329,960,928)$ & $(325,806,183)$ & $237,017,790$ \\
\hline Net Profit & $5,712,918,356$ & $3,573,818,036$ & $(2,513,813,108)$ & $8,471,889$ \\
\hline
\end{tabular}




\begin{tabular}{|c|c|c|c|c|}
\hline \multicolumn{5}{|l|}{$\begin{array}{l}\text { RS UMMI } \\
\text { Income Statement } \\
2018\end{array}$} \\
\hline Outpatient Income & $1,515,487,700$ & $\overline{1,231,002,900}$ & $1,643,386,400$ & $1,679,645,700$ \\
\hline Inpatient Income & $\mathbf{3 , 4 8 8 , 5 3 3 , 0 8 4}$ & $2,978,172,162$ & $3,772,578,143$ & $3,789,181,439$ \\
\hline Medical and Pharmaceutical Support Income & $2,784,682,145$ & $2,124,491,854$ & $2,695,328,230$ & $2,608,086,845$ \\
\hline The Ammount of Gross Operating Income & 7,788,702,929 & $6,333,666,916$ & $8,111,292,773$ & $8,076,913,984$ \\
\hline Difference in National Health Insurance & $(638,141,714)$ & $(445,322,702)$ & $(1,265,958,912)$ & $(1,376,066,162)$ \\
\hline The Ammount of Net Operating Income & $7,150,561,215$ & $5,888,344,214$ & $6,845,333,861$ & $6,700,847,822$ \\
\hline \multicolumn{5}{|l|}{ Direct Operating Expenses } \\
\hline Doctor's Fees & $1,849,500,774$ & $1,592,544,138$ & $1,891,919,415$ & $1,796,186,204$ \\
\hline Medical Support Burden & $132,295,559$ & $80,588,620$ & $155,553,399$ & $109,968,823$ \\
\hline Medical Operating Expenses & $208,169,571$ & $144,986,490$ & $201,098,593$ & $225,628,313$ \\
\hline Pharmaceutical Expenses & $1,141,582,249$ & $\mathbf{9 8 5 , 2 7 8 , 3 3 7}$ & $1,142,686,938$ & $985,653,385$ \\
\hline Other Direct Expenses & $105,526,320$ & $57,690,820$ & $124,386,542$ & $\mathbf{5 0 , 3 5 0 , 1 5 7}$ \\
\hline Total Direct Operating Expenses & $3,437,074,473$ & $2,861,088,405$ & $3,515,644,887$ & $3,167,786,882$ \\
\hline \multicolumn{5}{|l|}{ Indirect Operating Expenses } \\
\hline Staffing Burden & $1,731,588,115$ & $3,190,223,457$ & $1,851,116,678$ & $1,884,597,723$ \\
\hline Ajustments for The Difference in Stock & $(9,393,072)$ & $184,858,790$ & $39,192,109$ & $(8,726,574)$ \\
\hline Total Indirect Operating Expenses & $1,722,195,043$ & $3,375,082,247$ & $1,890,308,787$ & $1,875,871,149$ \\
\hline Total Operating Expenses & $5,159,269,516$ & $6,236,170,652$ & $5,405,953,674$ & $5,043,658,031$ \\
\hline Gross Profit & $1,991,291,699$ & $(347,826,438)$ & $1,439,380,187$ & $1,657,189,791$ \\
\hline \multicolumn{5}{|l|}{ Operating Expenses } \\
\hline General and Administrative Expenses & $186,988,824$ & $157,036,468$ & $203,475,896$ & $169,227,519$ \\
\hline Utility Expense & $115,701,217$ & $103,268,190$ & $95,950,964$ & $114,592,733$ \\
\hline Marketing Operational Expenses & $\mathbf{7 5 0 , 0 0 0}$ & 23,573,777 & $20,302,484$ & $52,406,735$ \\
\hline Maintenance and Repair Expenses & $41,457,227$ & $5,882,010$ & $68,376,744$ & $21,620,890$ \\
\hline Depreciation Expense & $384,342,775$ & $385,853,859$ & $389,759,420$ & $389,971,206$ \\
\hline Total Operating Expenses & $729,240,043$ & $675,614,304$ & $\mathbf{7 7 7 , 8 6 5 , 5 0 7}$ & $747,819,082$ \\
\hline \multicolumn{5}{|l|}{ Other Income / Expenses } \\
\hline Non Operating Income & $441,732,660$ & $32,299,331$ & $481,307,325$ & $240,723,649$ \\
\hline Non Operating Expenses & $371,231,279$ & $398,693,450$ & $353,274,885$ & $323,639,263$ \\
\hline The Ammount of Other Income/Expenses & $70,501,381$ & $(366,394,119)$ & $128,032,440$ & $(82,915,614)$ \\
\hline Net Profit & $1,332,553,037$ & $(\mathbf{1}, 389,834,861)$ & $789,547,120$ & $826,455,095$ \\
\hline
\end{tabular}


RS UMMI

Income Statement

2018

Account

Outpatient Income

Inpatient Income

Medical and Pharmaceutical Support Income

The Ammount of Gross Operating Income

Difference in National Health Insurance

The Ammount of Net Operating Income

Direct Operating Expenses

Doctor's Fees

Medical Support Burden

Medical Operating Expenses

Pharmaceutical Expenses

Other Direct Expenses

Total Direct Operating Expenses

Indirect Operating Expenses

Staffing Burden

Ajustments for The Difference in Stock

Total Indirect Operating Expenses

Total Operating Expenses

Gross Profit

Operating Expenses

General and Administrative Expenses

Utility Expense

Marketing Operational Expenses

Maintenance and Repair Expenses

Depreciation Expense

Total Operating Expenses

Other Income / Expenses

Non Operating Income

Non Operating Expenses

The Ammount of Other Income/Expenses

Net Profit

\begin{tabular}{|c|c|c|c|c|}
\hline Sep & Oct & Nov & Dec & Total \\
\hline $1,667,330,400$ & $1,940,264,500$ & $1,790,920,300$ & $1,746,677,200$ & $20,656,391,112$ \\
\hline $3,784,256,542$ & $4,282,358,554$ & 3,641,976,852 & $3,667,272,485$ & $44,788,671,169$ \\
\hline $2,279,969,497$ & $3,037,990,463$ & $2,894,469,694$ & $2,672,597,260$ & $31,579,135,094$ \\
\hline 7,731,556,439 & $9,260,613,517$ & $\mathbf{8 , 3 2 7 , 3 6 6 , 8 4 6}$ & $8,086,546,945$ & $\mathbf{9 7 , 0 2 4 , 1 9 7 , 3 7 5}$ \\
\hline$(1,273,306,343)$ & $(1,484,243,957)$ & $(1,142,951,407)$ & $(1,879,981,782)$ & $(15,422,605,167)$ \\
\hline $6,458,250,096$ & $7,776,369,560$ & $7,184,415,439$ & $6,206,565,163$ & $81,601,592,208$ \\
\hline 1,884,048,977 & $2,209,525,542$ & $863,680,967$ & 1,332,519,152 & $17,968,231,624$ \\
\hline $97,614,207$ & $148,447,873$ & $136,899,938$ & $138,293,419$ & $\mathbf{1 , 5 2 0 , 0 3 6 , 3 7 5}$ \\
\hline $216,535,119$ & $258,157,064$ & $222,405,501$ & 239,906,977 & 2,851,993,125 \\
\hline $985,023,537$ & $1,040,054,021$ & $1,001,856,770$ & $926,038,395$ & $12,215,288,393$ \\
\hline $64,950,236$ & $59,599,711$ & $63,237,682$ & $\mathbf{8 8 8 , 8 8 8 , 2 5 1}$ & $\mathbf{1 , 5 7 3 , 0 9 7 , 7 8 7}$ \\
\hline $3,248,172,076$ & $3,715,784,210$ & $2,288,080,858$ & $3,525,646,194$ & $36,128,647,304$ \\
\hline 1,769,572,895 & 1,822,668,882 & $\mathbf{1 , 8 9 4 , 4 8 1 , 5 6 1}$ & $2,049,792,776$ & $22,901,347,068$ \\
\hline$(17,843,333)$ & $5,469,171$ & $(1,264,251)$ & $(1,983,671)$ & $75,547,332$ \\
\hline $1,751,729,562$ & $\mathbf{1 , 8 2 8 , 1 3 8 , 0 5 3}$ & $1,893,217,310$ & $2,047,809,105$ & $22,976,894,400$ \\
\hline 4,999,901,638 & $5,543,922,263$ & $4,181,298,168$ & $5,573,455,299$ & $59,105,541,704$ \\
\hline $1,458,348,458$ & $2,232,447,297$ & $3,003,117,271$ & $633,109,864$ & $22,496,050,504$ \\
\hline $122,128,461$ & $187,385,361$ & $825,500,815$ & $(299,699,609)$ & $2,192,220,980$ \\
\hline $95,352,974$ & $110,782,940$ & $116,997,898$ & $109,234,547$ & $1,265,365,194$ \\
\hline $12,777,732$ & $11,078,180$ & $11,930,751$ & $68,646,074$ & $313,567,228$ \\
\hline $74,126,998$ & $37,176,992$ & $84,746,573$ & $69,023,752$ & $531,524,438$ \\
\hline $388,655,211$ & $389,317,111$ & $379,954,116$ & $404,462,585$ & $4,757,583,403$ \\
\hline $693,041,376$ & $735,740,584$ & $1,419,130,153$ & $351,667,349$ & $9,060,261,243$ \\
\hline 201,510,887 & $628,154,056$ & $(755,699,399)$ & $(273,959,919)$ & $1,686,381,348$ \\
\hline $316,049,342$ & $\mathbf{3 , 8 6 5 , 8 7 7 , 5 1 9}$ & $1,793,176,268$ & $(4,354,066,226)$ & $4,475,642,894$ \\
\hline$(114,538,455)$ & $(3,237,723,463)$ & $(2,548,875,667)$ & $4,080,106,307$ & $(2,789,261,546)$ \\
\hline $650,768,627$ & $(1,741,016,750)$ & $(964,888,549)$ & $4,361,548,823$ & $10,646,527,715$ \\
\hline
\end{tabular}

\subsection{Analysis of Income}

The following is a recapitulation of Ummi's hospital revenue for outpatient and inpatient services for both general patients and National Health Insurance patients from 2016, 2017 and 2018: patents

\begin{tabular}{|c|c|c|c|c|c|}
\hline \multirow[b]{2}{*}{ MONTH } & \multicolumn{2}{|c|}{ OUTPATIENT INCOME } & \multicolumn{2}{|c|}{ INPATIENT INCOME } & \multirow[b]{2}{*}{ TOTAL } \\
\hline & $\begin{array}{c}\text { GENERAL } \\
\text { OUTPATIENT } \\
\text { CARE }\end{array}$ & $\begin{array}{c}\text { OUTPATIENT } \\
\text { NHI }\end{array}$ & $\begin{array}{c}\text { GENERAL } \\
\text { HOSPITALIZATION }\end{array}$ & $\begin{array}{l}\text { INPATIENT } \\
\text { NHI }\end{array}$ & \\
\hline JANUARY & $572,991,621$ & $830,668,222$ & $1,014,271,220$ & $1,578,939,413$ & $3,996,870,476$ \\
\hline FEBRUARY & $722,373,286$ & $630,309,566$ & $962,298,952$ & $1,513,576,877$ & $3,828,558,681$ \\
\hline MARCH & $752,862,574$ & $928,546,180$ & $1,475,665,659$ & $1,694,844,455$ & $4,851,918,868$ \\
\hline APRIL & $703,801,904$ & $1,115,482,741$ & $1,146,102,507$ & $1,813,665,052$ & $4,779,052,204$ \\
\hline MAY & $835,071,668$ & $1,297,994,452$ & $1,109,887,747$ & $1,796,476,734$ & $5,039,430,601$ \\
\hline JUNE & $674,282,192$ & $983,162,096$ & $1,135,056,415$ & $1,797,671,018$ & $4,590,171,721$ \\
\hline JULY & $719,407,947$ & $991,632,422$ & $1,041,932,773$ & $1,662,339,932$ & $\mathbf{4 , 4 1 5 , 3 1 3 , 0 7 4}$ \\
\hline AUGUST & $820,572,344$ & $1,316,302,879$ & $\mathbf{1 , 0 4 4 , 1 9 7 , 8 5 6}$ & $1,934,046,207$ & $5,115,119,286$ \\
\hline SEPTEMBER & $613,470,516$ & $1,418,783,538$ & $1,122,410,207$ & $2,018,904,454$ & $5,173,568,715$ \\
\hline OCTOBER & $1,013,237,863$ & $1,186,613,931$ & $1,293,327,732$ & $1,831,078,474$ & $5,324,258,000$ \\
\hline NOVEMBER & $\mathbf{8 3 5}, \mathbf{5 4 1 , 7 7 7}$ & $1,271,833,434$ & $1,016,924,948$ & $1,954,928,691$ & $5,079,228,850$ \\
\hline DECEMBER & 930,023,366 & $1,207,687,500$ & $941,822,202$ & $1,988,127,334$ & $5,067,660,402$ \\
\hline TOTAL & $9,193,637,058$ & $13,179,016,961$ & $13,303,898,218$ & $21,584,598,641$ & $57,261,150,878$ \\
\hline
\end{tabular}


Muhammad Zafrullah \& Hadri Mulya., Sch J Econ Bus Manag, Apr, 2021; 8(4): 110-135

REVENUE IN 2017

\begin{tabular}{|l|r|r|r|r|r|}
\hline \multirow{4}{*}{ MONTH } & \multicolumn{2}{|c|}{ OUTPATIENT INCOME } & \multicolumn{2}{|c|}{ INPATIENT INCOME } & \multirow{2}{*}{ TOTAL } \\
\cline { 2 - 5 } & $\begin{array}{c}\text { GENERAL } \\
\text { OUTPATIENT } \\
\text { CARE }\end{array}$ & $\begin{array}{c}\text { OUTPATIENT } \\
\text { NHI }\end{array}$ & $\begin{array}{c}\text { GENERAL } \\
\text { HOSPITALIZATION }\end{array}$ & $\begin{array}{c}\text { INPATIENT } \\
\text { NHI }\end{array}$ & \\
\hline JANUARY & $\mathbf{8 9 1 , 8 0 4 , 3 5 7}$ & $\mathbf{1 , 1 5 4 , 5 7 4 , 4 8 6}$ & $\mathbf{9 2 8 , 6 4 4 , 3 2 8}$ & $\mathbf{2 , 5 9 3 , 4 3 0 , 6 2 5}$ & $\mathbf{5 , 5 6 8 , 4 5 3 , 7 9 6}$ \\
\hline FEBRUARY & $\mathbf{8 7 9 , 0 9 3 , 0 8 2}$ & $\mathbf{1 , 1 4 7 , 7 0 0 , 9 5 4}$ & $\mathbf{1 , 0 3 6 , 3 5 5 , 8 8 5}$ & $\mathbf{2 , 5 7 6 , 8 8 6 , 2 5 1}$ & $\mathbf{5 , 6 4 0 , 0 3 6 , 1 7 2}$ \\
\hline MARCH & $\mathbf{7 6 3 , 0 2 3 , 6 1 7}$ & $\mathbf{1 , 6 1 6 , 8 8 7 , 2 4 8}$ & $\mathbf{9 9 3 , 0 3 3 , 3 7 5}$ & $\mathbf{3 , 0 7 1 , 7 0 1 , 6 6 8}$ & $\mathbf{6 , 4 4 4 , 6 4 5 , 9 0 8}$ \\
\hline APRIL & $\mathbf{8 6 0 , 3 0 7 , 3 8 7}$ & $\mathbf{1 , 4 0 4 , 0 1 7 , 3 0 9}$ & $\mathbf{8 2 5 , 1 9 2 , 3 7 3}$ & $\mathbf{3 , 0 3 8 , 1 8 7 , 0 9 1}$ & $\mathbf{6 , 1 2 7 , 7 0 4 , 1 6 0}$ \\
\hline MAY & $\mathbf{6 1 8 , 0 6 7 , 2 6 3}$ & $\mathbf{1 , 7 2 1 , 6 3 4 , 6 3 7}$ & $\mathbf{9 4 5 , 8 4 8 , 8 5 3}$ & $\mathbf{3 , 3 5 4 , 7 1 6 , 1 3 6}$ & $\mathbf{6 , 6 4 0 , 2 6 6 , 8 8 9}$ \\
\hline JUNE & $\mathbf{7 1 4 , 4 2 8 , 1 5 6}$ & $\mathbf{1 , 2 7 8 , 2 2 5 , 1 4 1}$ & $\mathbf{8 7 5 , 7 1 9 , 8 7 8}$ & $\mathbf{2 , 6 3 0 , 5 2 3 , 5 8 0}$ & $\mathbf{5 , 4 9 8 , 8 9 6 , 7 5 5}$ \\
\hline JULY & $\mathbf{7 5 5 , 8 2 7 , 4 4 6}$ & $\mathbf{1 , 7 5 9 , 5 4 7 , 0 6 4}$ & $\mathbf{9 3 3 , 3 2 3 , 4 4 3}$ & $\mathbf{3 , 8 0 1 , 4 0 5 , 4 9 4}$ & $\mathbf{7 , 2 5 0 , 1 0 3 , 4 4 7}$ \\
\hline AUGUST & $\mathbf{6 4 5 , 9 3 0 , 3 4 5}$ & $\mathbf{2 , 0 3 2 , 6 8 5 , 4 7 9}$ & $\mathbf{8 9 0 , 8 1 1 , 3 6 4}$ & $\mathbf{4 , 2 2 8 , 4 4 5 , 5 7 2}$ & $\mathbf{7 , 7 9 7 , 8 7 2 , 7 6 0}$ \\
\hline SEPTEMBER & $\mathbf{6 9 2 , 9 0 4 , 6 5 4}$ & $\mathbf{1 , 9 2 1 , 6 9 6 , 8 5 1}$ & $\mathbf{7 6 0 , 6 5 0 , 8 9 5}$ & $\mathbf{3 , 8 1 7 , 0 9 5 , 2 1 8}$ & $\mathbf{7 , 1 9 2 , 3 4 7 , 6 1 8}$ \\
\hline OCTOBER & $\mathbf{9 1 8 , 9 2 4 , 4 0 3}$ & $\mathbf{2 , 1 7 0 , 3 0 4 , 0 7 8}$ & $\mathbf{9 7 0 , 5 4 2 , 0 9 5}$ & $\mathbf{3 , 9 5 8 , 1 0 0 , 4 3 4}$ & $\mathbf{8 , 0 1 7 , 8 7 1 , 0 1 0}$ \\
\hline NOVEMBER & $\mathbf{6 2 1 , 8 7 5 , 4 6 2}$ & $\mathbf{2 , 2 7 2 , 2 8 6 , 8 1 7}$ & $\mathbf{1 , 1 0 3 , 3 9 0 , 3 5 8}$ & $\mathbf{4 , 1 8 7 , 4 9 3 , 8 4 2}$ & $\mathbf{8 , 1 8 5 , 0 4 6 , 4 7 9}$ \\
\hline DECEMBER & $\mathbf{7 7 4 , 9 2 5 , 5 5 2}$ & $\mathbf{2 , 1 4 9 , 4 0 4 , 9 0 4}$ & $\mathbf{9 0 7 , 5 4 5 , 6 3 4}$ & $\mathbf{4 , 8 3 8 , 5 3 5 , 3 3 0}$ & $\mathbf{8 , 6 7 0 , 4 1 1 , 4 2 0}$ \\
\hline \multicolumn{1}{|c|}{ TOTAL } & $\mathbf{9 , 1 3 7 , 1 1 1 , 7 2 4}$ & $\mathbf{2 0 , 6 2 8 , 9 6 4 , 9 6 8}$ & $\mathbf{1 1 , 1 7 1 , 0 5 8 , 4 8 1}$ & $\mathbf{4 2 , 0 9 6 , 5 2 1 , 2 4 1}$ & $\mathbf{8 3 , 0 3 3 , 6 5 6 , 4 1 4}$ \\
\hline
\end{tabular}

REVENUE IN 2018

\begin{tabular}{|l|r|r|r|r|r|}
\hline \multirow{2}{*}{ MONTH } & \multicolumn{2}{|c|}{ OUTPATIENT INCOME } & \multicolumn{2}{c|}{ INPATIENT INCOME } & \multirow{2}{*}{ TOTAL } \\
\cline { 2 - 5 } & $\begin{array}{c}\text { GENERAL } \\
\text { OUTPATIENT } \\
\text { CARE }\end{array}$ & $\begin{array}{c}\text { OUTPATIENT } \\
\text { NHI }\end{array}$ & $\begin{array}{c}\text { GENERAL } \\
\text { HOSPITALIZATION }\end{array}$ & $\begin{array}{c}\text { INPATIENT } \\
\text { NHI }\end{array}$ & \\
\hline JANUARY & $\mathbf{1 , 1 5 6 , 9 0 3 , 6 0 9}$ & $\mathbf{2 , 8 3 2 , 5 9 8 , 5 9 1}$ & $\mathbf{9 3 4 , 5 1 5 , 8 9 5}$ & $\mathbf{5 , 5 9 3 , 0 8 5 , 9 7 4}$ & $\mathbf{1 0 , 5 1 7 , 1 0 4 , 0 6 9}$ \\
\hline FEBRUARY & $\mathbf{6 8 4 , 3 0 6 , 6 2 0}$ & $\mathbf{2 , 5 1 9 , 7 5 2 , 8 9 6}$ & $\mathbf{9 1 4 , 2 1 2 , 2 0 8}$ & $\mathbf{3 , 9 2 1 , 4 3 6 , 7 2 5}$ & $\mathbf{8 , 0 3 9 , 7 0 8 , 4 4 9}$ \\
\hline MARCH & $\mathbf{7 5 6 , 6 1 4 , 5 7 8}$ & $\mathbf{1 , 6 7 0 , 7 8 2 , 7 6 6}$ & $\mathbf{9 7 7 , 3 5 5 , 0 3 3}$ & $\mathbf{4 , 0 6 6 , 2 8 1 , 9 2 0}$ & $\mathbf{7 , 4 7 1 , 0 3 4 , 2 9 7}$ \\
\hline APRIL & $\mathbf{1 , 0 7 8 , 6 8 0 , 7 8 6}$ & $\mathbf{1 , 6 6 7 , 3 4 9 , 2 2 9}$ & $\mathbf{8 4 5 , 4 8 5 , 6 0 4}$ & $\mathbf{3 , 6 8 8 , 1 7 4 , 5 9 2}$ & $\mathbf{7 , 2 7 9 , 6 9 0 , 2 1 1}$ \\
\hline MAY & $\mathbf{1 , 1 4 2 , 4 6 3 , 5 5 4}$ & $\mathbf{1 , 7 9 5 , 6 5 6 , 5 0 6}$ & $\mathbf{1 , 1 0 4 , 6 5 8 , 5 6 0}$ & $\mathbf{3 , 7 4 5 , 9 2 4 , 3 0 9}$ & $\mathbf{7 , 7 8 8 , 7 0 2 , 9 2 9}$ \\
\hline JUNE & $\mathbf{4 3 2 , 7 0 6 , 8 1 9}$ & $\mathbf{1 , 4 1 0 , 8 5 5 , 6 4 0}$ & $\mathbf{9 6 9 , 3 9 4 , 0 7 1}$ & $\mathbf{3 , 5 2 0 , 7 1 0 , 3 8 6}$ & $\mathbf{6 , 3 3 3 , 6 6 6 , 9 1 6}$ \\
\hline JULY & $\mathbf{7 6 6 , 4 9 9 , 9 6 4}$ & $\mathbf{1 , 9 6 5 , 3 9 4 , 4 1 5}$ & $\mathbf{1 , 1 4 5 , 5 0 6 , 0 1 6}$ & $\mathbf{4 , 2 3 3 , 8 9 2 , 3 7 8}$ & $\mathbf{8 , 1 1 1 , 2 9 2 , 7 7 3}$ \\
\hline AUGUST & $\mathbf{9 9 6 , 2 7 5 , 3 1 2}$ & $\mathbf{1 , 9 0 2 , 0 1 1 , 4 2 1}$ & $\mathbf{8 6 4 , 2 3 0 , 7 4 1}$ & $\mathbf{4 , 3 1 4 , 3 9 6 , 5 1 0}$ & $\mathbf{8 , 0 7 6 , 9 1 3 , 9 8 4}$ \\
\hline SEPTEMBER & $\mathbf{5 7 1 , 4 7 0 , 6 8 6}$ & $\mathbf{1 , 9 4 4 , 6 4 3 , 7 9 9}$ & $\mathbf{1 , 1 5 1 , 8 8 3 , 6 6 4}$ & $\mathbf{4 , 0 6 3 , 5 5 8 , 2 9 0}$ & $\mathbf{7 , 7 3 1 , 5 5 6 , 4 3 9}$ \\
\hline OCTOBER & $\mathbf{7 8 6 , 1 6 2 , 7 3 3}$ & $\mathbf{2 , 4 2 8 , 5 7 0 , 4 8 3}$ & $\mathbf{1 , 3 8 3 , 3 4 0 , 9 8 6}$ & $\mathbf{4 , 6 6 2 , 5 3 9 , 3 1 5}$ & $\mathbf{9 , 2 6 0 , 6 1 3 , 5 1 7}$ \\
\hline NOVEMBER & $\mathbf{6 6 7 , 7 5 4 , 5 8 4}$ & $\mathbf{2 , 3 1 1 , 9 4 3 , 9 2 2}$ & $\mathbf{1 , 0 2 1 , 5 1 3 , 3 8 7}$ & $\mathbf{4 , 3 2 6 , 1 5 4 , 9 5 3}$ & $\mathbf{8 , 3 2 7 , 3 6 6 , 8 4 6}$ \\
\hline DECEMBER & $\mathbf{8 0 1 , 6 4 5 , 5 6 1}$ & $\mathbf{2 , 2 3 7 , 2 4 9 , 4 7 0}$ & $\mathbf{1 , 0 3 7 , 1 1 8 , 3 8 7}$ & $\mathbf{4 , 0 1 0 , 5 3 3 , 5 2 7}$ & $\mathbf{8 , 0 8 6 , 5 4 6 , 9 4 5}$ \\
\hline \multicolumn{1}{|c|}{ TOTAL } & $\mathbf{9 , 8 4 1 , 4 8 4 , 8 0 6}$ & $\mathbf{2 4 , 6 8 6 , 8 0 9 , 1 3 8}$ & $\mathbf{1 2 , 3 4 9 , 2 1 4 , 5 5 2}$ & $\mathbf{5 0 , 1 4 6 , 6 8 8 , 8 7 9}$ & $\mathbf{9 7 , 0 2 4 , 1 9 7 , 3 7 5}$ \\
\hline
\end{tabular}

The contribution of Ummi's hospital income between general patients and National Health Insurance patients, in 2016 amounted to $39 \%$ for general patients and $61 \%$ for National Health Insurance patients, in 2017 it was $24 \%$ for general patients and $76 \%$ for National Health Insurance patients, then in 2018 it was $23 \%$ for general patients. and $77 \%$ of National Health Insurance patients. In recording its income transactions, the Bogor UMMI hospital uses the Accrual Basis method in accordance with statement of accounting standard number 23, where income is recognized when billing or invoices for patients are issued.

\subsubsection{Analysis of Hospital Income from General Patients}

Income from general patients is income from services for company insurance patients, insurance coverage, dependent patients from municipal and district health offices and private patients or cash. Revenue recognition for general patients using the existing hospital rates in the master hospital rates.

\subsubsection{Analysis of Hospital Income from National Health Insurance Patients}

To recognize hospital income from National Health Insurance patients, use the regular rate value from the current hospital master rate and that value is included in the billing or invoice for National Health Insurance patients. To process claims or billing for National Health Insurance patients to National Health Insurance then the income value is re-coding based on the INA-CBG's rates from National Health Insurance before all documents from the service are submitted to the National Health Insurance office. The difference in the recorded value of National Health Insurance patient income based on the regular rate value of the hospital's master rate compared to the INA-CBG's National Health Insurance rate coding value is recorded in the National Health Insurance Difference account in the Income Statement. The following is the value of the difference in recording National Health Insurance patient income based on the regular value of the hospital master rate with the INA-CBG's National Health Insurance rate coding value from 2016, 2017 and 2018: 


\begin{tabular}{|c|c|c|c|}
\hline \multicolumn{4}{|c|}{$\begin{array}{l}\text { RS UMMI } \\
\text { COMPARISON OF HOSPITAL RATES WITH INA-CBG'S NHI RATES IN } 2016\end{array}$} \\
\hline $\begin{array}{c}\text { MONTH } \\
\text { OF SERVICE }\end{array}$ & $\begin{array}{c}\text { NET NHI } \\
\text { (HOSPITAL RATES) }\end{array}$ & $\begin{array}{c}\text { NET NHI } \\
\text { (INA-CBG's RATES) }\end{array}$ & DIFFERENCE \\
\hline JANUARY & $2,409,607,635$ & $2,052,543,500$ & $357,064,135$ \\
\hline FEBRUARY & $2,143,886,443$ & $1,822,963,100$ & $320,923,343$ \\
\hline MARCH & $2,623,390,635$ & $2,215,793,200$ & $407,597,435$ \\
\hline APRIL & $2,929,147,793$ & $2,418,718,900$ & $510,428,893$ \\
\hline MAY & $3,094,471,186$ & $2,258,666,700$ & $835,804,486$ \\
\hline JUNE & $2,780,833,114$ & $1,896,444,600$ & $884,388,514$ \\
\hline JULY & $2,653,972,354$ & $1,867,829,600$ & $786,142,754$ \\
\hline AUGUST & $3,250,349,086$ & $2,392,866,900$ & $857,482,186$ \\
\hline SEPTEMBER & $3,437,687,992$ & $2,626,773,700$ & $810,914,292$ \\
\hline OCTOBER & $3,017,692,405$ & $2,522,991,500$ & $494,700,905$ \\
\hline NOVEMBER & $3,226,762,125$ & $2,680,209,200$ & $546,552,925$ \\
\hline DECEMBER & $3,195,814,834$ & $2,685,304,900$ & $510,509,934$ \\
\hline TOTAL & $34,763,615,602$ & $27,441,105,800$ & $7,322,509,802$ \\
\hline
\end{tabular}

\begin{tabular}{|c|c|c|c|}
\hline \multicolumn{4}{|c|}{$\begin{array}{l}\text { RS UMMI } \\
\text { COMPARISON OF HOSPITAL RATES WITH INA-CBG'S NHI RATES IN } 2017\end{array}$} \\
\hline $\begin{array}{c}\text { MONTH } \\
\text { OF SERVICE }\end{array}$ & $\begin{array}{c}\text { NET NHI } \\
\text { (HOSPITAL RATES) }\end{array}$ & $\begin{array}{c}\text { NET NHI } \\
\text { (INA-CBG's RATES) }\end{array}$ & DIFFERENCE \\
\hline JANUARY & $3,748,005,111$ & $2,785,557,100$ & $962,448,011$ \\
\hline FEBRUARY & $3,724,587,205$ & $2,749,624,100$ & $974,963,105$ \\
\hline MARCH & $4,688,588,916$ & $3,314,097,800$ & $1,374,491,116$ \\
\hline APRIL & $4,442,204,400$ & $3,075,547,500$ & $1,366,656,900$ \\
\hline MAY & $5,076,350,773$ & $3,531,539,100$ & $1,544,811,673$ \\
\hline JUNE & $3,908,748,721$ & $2,790,766,900$ & $1,117,981,821$ \\
\hline JULY & $5,560,952,558$ & $3,625,730,800$ & $1,935,221,758$ \\
\hline AUGUST & $6,261,131,051$ & $3,918,679,200$ & $2,342,451,851$ \\
\hline SEPTEMBER & $5,738,792,069$ & $3,494,820,400$ & $2,243,971,669$ \\
\hline OCTOBER & $6,128,404,512$ & $3,929,114,100$ & $2,199,290,412$ \\
\hline NOVEMBER & $6,459,780,659$ & $4,008,454,900$ & $2,451,325,759$ \\
\hline DECEMBER & $6,987,940,234$ & $4,421,173,400$ & $2,566,766,834$ \\
\hline TOTAL & $62,725,486,209$ & $41,645,105,300$ & $21,080,380,909$ \\
\hline
\end{tabular}

\begin{tabular}{|l|r|r|r|}
\hline \multicolumn{5}{|l|}{ RS UMMI } \\
COMPARISON OF HOSPITAL RATES WITH INA-CBG's NHI RATES IN 2018 \\
\begin{tabular}{|l|c|r|} 
MONTH \\
OF SERVICE
\end{tabular} & $\begin{array}{c}\text { NET NHI NHI } \\
\text { (HOSPITAL RATES) }\end{array}$ & DIFFERENCE \\
\hline JANUARY & $\mathbf{8 , 4 2 5 , 6 8 4 , 5 6 5}$ & $\mathbf{5 , 3 2 8 , 8 8 1 , 3 0 0}$ & $\mathbf{3 , 0 9 6 , 8 0 3 , 2 6 5}$ \\
\hline FEBRUARY & $\mathbf{6 , 4 4 1 , 1 8 9 , 6 2 1}$ & $\mathbf{4 , 1 0 3 , 7 6 3 , 6 0 0}$ & $\mathbf{2 , 3 3 7 , 4 2 6 , 0 2 1}$ \\
\hline MARCH & $\mathbf{5 , 7 3 7 , 0 6 4 , 6 8 6}$ & $\mathbf{4 , 4 1 1 , 7 8 9 , 1 0 0}$ & $\mathbf{1 , 3 2 5 , 2 7 5 , 5 8 6}$ \\
\hline APRIL & $\mathbf{5 , 3 5 5 , 5 2 3 , 8 2 1}$ & $\mathbf{4 , 5 1 8 , 8 8 6 , 1 0 0}$ & $\mathbf{8 3 6 , 6 3 7 , 7 2 1}$ \\
\hline MAY & $\mathbf{5 , 5 4 1 , 5 8 0 , 8 1 5}$ & $\mathbf{4 , 7 0 6 , 6 1 8 , 3 0 0}$ & $\mathbf{8 3 4 , 9 6 2 , 5 1 5}$ \\
\hline JUNE & $\mathbf{4 , 9 3 1 , 5 6 6 , 0 2 6}$ & $\mathbf{4 , 1 0 9 , 7 9 3 , 0 0 0}$ & $\mathbf{8 2 1 , 7 7 3 , 0 2 6}$ \\
\hline JULY & $\mathbf{6 , 1 9 9 , 2 8 6 , 7 9 3}$ & $\mathbf{5 , 1 1 6 , 5 7 7 , 1 0 0}$ & $\mathbf{1 , 0 8 2 , 7 0 9 , 6 9 3}$ \\
\hline AUGUST & $\mathbf{6 , 2 1 6 , 4 0 7 , 9 3 1}$ & $\mathbf{4 , 8 8 5 , 5 8 2 , 5 0 0}$ & $\mathbf{1 , 3 3 0 , 8 2 5 , 4 3 1}$ \\
\hline SEPTEMBER & $\mathbf{6 , 0 0 8 , 2 0 2 , 0 8 9}$ & $\mathbf{4 , 9 3 0 , 0 9 3 , 0 0 0}$ & $\mathbf{1 , 0 7 8 , 1 0 9 , 0 8 9}$ \\
\hline OCTOBER & $\mathbf{7 , 0 9 1 , 1 0 9 , 7 9 8}$ & $\mathbf{5 , 6 6 3 , 1 8 3 , 7 0 0}$ & $\mathbf{1 , 4 2 7 , 9 2 6 , 0 9 8}$ \\
\hline NOVEMBER & $\mathbf{6 , 6 3 8 , 0 9 8 , 8 7 5}$ & $\mathbf{5 , 2 4 2 , 8 6 6 , 2 0 0}$ & $\mathbf{1 , 3 9 5 , 2 3 2 , 6 7 5}$ \\
\hline DECEMBER & $\mathbf{6 , 2 4 7 , 7 8 2 , 9 9 7}$ & $\mathbf{5 , 2 0 7 , 5 4 7 , 3 0 0}$ & $\mathbf{1 , 0 4 0 , 2 3 5 , 6 9 7}$ \\
\hline \multicolumn{1}{|c|}{ TOTAL } & $\mathbf{7 4 , 8 3 3 , 4 9 8 , 0 1 7}$ & $\mathbf{5 8 , 2 2 5 , 5 8 1 , 2 0 0}$ & $\mathbf{1 6 , 6 0 7 , 9 1 6 , 8 1 7}$ \\
\hline
\end{tabular}

The value of the difference between National Health Insurance and the total value of recorded income for National Health Insurance patients with the two treatments, for 2016 amounting to Rp. 7,322,509,802, in 2017 amounting to Rp. 21,080,380,909, - and in 2018 amounting to Rp. -. The following is the value of the difference in recording National Health Insurance patient income based on a value of $70 \%$ from the hospital master rate with the INA-CBG's National Health Insurance rate coding value for outpatient and inpatient services from 2016, 2017 and 2018: 


\begin{tabular}{|c|c|c|c|}
\hline \multicolumn{4}{|c|}{$\begin{array}{l}\text { RS UMMI } \\
\text { COMPARISON OF HOSPITAL RATES WITH INA-CBG'S NHI RATES } \\
\text { OUTPATIENT IN } 2016\end{array}$} \\
\hline $\begin{array}{c}\text { MONTH } \\
\text { OF SERVICE } \\
\end{array}$ & $\begin{array}{c}\text { NET NHI } \\
\text { (HOSPITAL RATES) } \\
\end{array}$ & $\begin{array}{c}\text { NET NHI } \\
\text { (INA-CBG'S RATES) }\end{array}$ & DIFFERENCE \\
\hline JANUARY & $830,668,222$ & $852,929,400$ & $(22,261,178)$ \\
\hline FEBRUARY & $630,309,566$ & $667,065,000$ & $(36,755,434)$ \\
\hline MARCH & $928,546,180$ & $915,534,900$ & $13,011,280$ \\
\hline APRIL & $1,115,482,741$ & $1,107,804,600$ & $7,678,141$ \\
\hline MAY & $1,297,994,452$ & $1,082,790,200$ & $215,204,252$ \\
\hline JUNE & $983,162,096$ & $784,321,100$ & $198,840,996$ \\
\hline JULY & $991,632,422$ & $866,288,800$ & $125,343,622$ \\
\hline AUGUST & $1,316,302,879$ & $1,116,922,100$ & $199,380,779$ \\
\hline SEPTEMBER & $1,418,783,538$ & $1,204,405,700$ & $214,377,838$ \\
\hline OCTOBER & $1,186,613,931$ & $1,079,216,600$ & $107,397,331$ \\
\hline NOVEMBER & $1,271,833,434$ & $1,127,170,100$ & $144,663,334$ \\
\hline DECEMBER & $1,207,687,500$ & $1,116,964,300$ & $90,723,200$ \\
\hline TOTAL & $13,179,016,961$ & $11,921,412,800$ & $1,257,604,161$ \\
\hline
\end{tabular}

\begin{tabular}{|c|c|c|c|}
\hline \multicolumn{4}{|c|}{$\begin{array}{l}\text { RS UMMI } \\
\text { COMPARISON OF HOSPITAL RATES WITH INA-CBG'S NHI RATES } \\
\text { OUTPATIENT IN } 2017\end{array}$} \\
\hline $\begin{array}{c}\text { MONTH } \\
\text { OF SERVICE } \\
\end{array}$ & $\begin{array}{c}\text { NET NHI } \\
\text { (HOSPITAL RATES) } \\
\end{array}$ & $\begin{array}{c}\text { NET NHI } \\
\text { (INA-CBG's RATES) } \\
\end{array}$ & DIFFERENCE \\
\hline JANUARY & $1,154,574,486$ & $1,010,004,000$ & $144,570,486$ \\
\hline FEBRUARY & $1,147,700,954$ & $1,011,342,800$ & $136,358,154$ \\
\hline MARCH & $1,616,887,248$ & $1,337,145,800$ & $279,741,448$ \\
\hline APRIL & $1,404,017,309$ & $1,167,808,200$ & $236,209,109$ \\
\hline MAY & $1,721,634,637$ & $1,309,481,800$ & $412,152,837$ \\
\hline JUNE & $1,278,225,141$ & $1,024,320,400$ & $253,904,741$ \\
\hline JULY & $1,759,547,064$ & $1,280,177,200$ & $479,369,864$ \\
\hline AUGUST & $2,032,685,479$ & $1,490,134,100$ & $542,551,379$ \\
\hline SEPTEMBER & $1,921,696,851$ & $1,393,114,500$ & $528,582,351$ \\
\hline OCTOBER & $2,170,304,078$ & $1,598,513,200$ & $\mathbf{5 7 1 , 7 9 0 , 8 7 8}$ \\
\hline NOVEMBER & $2,272,286,817$ & $1,664,802,300$ & $607,484,517$ \\
\hline DECEMBER & $2,149,404,904$ & $1,555,426,100$ & $593,978,804$ \\
\hline $\begin{array}{l}\text { TOTAL } \\
\end{array}$ & $20,628,964,968$ & $15,842,270,400$ & $4,786,694,568$ \\
\hline
\end{tabular}

\begin{tabular}{|c|c|c|c|}
\hline \multicolumn{4}{|c|}{$\begin{array}{l}\text { RS UMMI } \\
\text { COMPARISON OF HOSPITAL RATES WITH INA-CBG'S NHI RATES } \\
\text { OUTPATIENT IN } 2018\end{array}$} \\
\hline $\begin{array}{c}\text { MONTH } \\
\text { OF SERVICE }\end{array}$ & $\begin{array}{c}\text { NET NHI } \\
\text { (HOSPITAL RATES) }\end{array}$ & $\begin{array}{c}\text { NET NHI } \\
\text { (INA-CBG's RATES) }\end{array}$ & DIFFERENCE \\
\hline JANUARY & $2,832,598,591$ & $1,964,500,000$ & $868,098,591$ \\
\hline FEBRUARY & $2,519,752,896$ & $1,724,272,100$ & $795,480,796$ \\
\hline MARCH & $1,670,782,766$ & $1,693,935,500$ & $(23,152,734)$ \\
\hline APRIL & $1,667,349,229$ & $1,801,463,600$ & $(134,114,371)$ \\
\hline MAY & $1,795,656,506$ & $1,908,835,100$ & $(113,178,594)$ \\
\hline JUNE & $1,410,855,640$ & $1,500,798,900$ & $(89,943,260)$ \\
\hline JULY & $1,965,394,415$ & $2,071,208,000$ & $(105,813,585)$ \\
\hline AUGUST & $1,902,011,421$ & $2,008,402,800$ & $(106,391,379)$ \\
\hline SEPTEMBER & $1,944,643,799$ & $2,083,792,900$ & $(139,149,101)$ \\
\hline OCTOBER & $2,428,570,483$ & $2,548,970,400$ & $(120,399,917)$ \\
\hline NOVEMBER & $2,311,943,922$ & $2,434,649,900$ & $(122,705,978)$ \\
\hline DECEMBER & $2,237,249,470$ & $2,362,987,000$ & $(125,737,530)$ \\
\hline TOTAL & $24,686,809,138$ & $24,103,816,200$ & $582,992,938$ \\
\hline
\end{tabular}

For National Health Insurance patients for outpatient services, the difference in National Health Insurance recorded is in 2016 amounting to $\mathrm{Rp}$.
$1,257,604,161, \quad$ - in 2017 amounting to Rp. $4,786,694,568$, - and in 2018 amounting to Rp. $582,992,938,-$. 
RS UMMI

COMPARISON OF HOSPITAL RATES WITH INA-CBG'S NHI RATES

INPATIENTS IN 2016

\begin{tabular}{|l|r|r|r|}
\hline $\begin{array}{c}\text { MONTH } \\
\text { OF SER VICE }\end{array}$ & $\begin{array}{c}\text { NET NHI } \\
\text { (HOSPITAL RATES) }\end{array}$ & $\begin{array}{c}\text { NET NHI } \\
\text { (INA-CBG's RATES) }\end{array}$ & \multicolumn{1}{c|}{ DIFFERENCE } \\
\hline JANUARY & $\mathbf{1 , 5 7 8 , 9 3 9 , 4 1 3}$ & $\mathbf{1 , 1 9 9 , 6 1 4 , 1 0 0}$ & $\mathbf{3 7 9 , 3 2 5 , 3 1 3}$ \\
\hline FEBRUARY & $\mathbf{1 , 5 1 3 , 5 7 6 , 8 7 7}$ & $\mathbf{1 , 1 5 5 , 8 9 8 , 1 0 0}$ & $\mathbf{3 5 7 , 6 7 8 , 7 7 7}$ \\
\hline MARCH & $\mathbf{1 , 6 9 4 , 8 4 4 , 4 5 5}$ & $\mathbf{1 , 3 0 0 , 2 5 8 , 3 0 0}$ & $\mathbf{3 9 4 , 5 8 6 , 1 5 5}$ \\
\hline APRIL & $\mathbf{1 , 8 1 3 , 6 6 5 , 0 5 2}$ & $\mathbf{1 , 3 1 0 , 9 1 4 , 3 0 0}$ & $\mathbf{5 0 2 , 7 5 0 , 7 5 2}$ \\
\hline MAY & $\mathbf{1 , 7 9 6 , 4 7 6 , 7 3 4}$ & $\mathbf{1 , 1 7 5 , 8 7 6 , 5 0 0}$ & $\mathbf{6 2 0 , 6 0 0 , 2 3 4}$ \\
\hline JUNE & $\mathbf{1 , 7 9 7 , 6 7 1 , 0 1 8}$ & $\mathbf{1 , 1 1 2 , 1 2 3 , 5 0 0}$ & $\mathbf{6 8 5 , 5 4 7 , 5 1 8}$ \\
\hline JULY & $\mathbf{1 , 6 6 2 , 3 3 9 , 9 3 2}$ & $\mathbf{1 , 0 0 1 , 5 4 0 , 8 0 0}$ & $\mathbf{6 6 0 , 7 9 9 , 1 3 2}$ \\
\hline AUGUST & $\mathbf{1 , 9 3 4 , 0 4 6 , 2 0 7}$ & $\mathbf{1 , 2 7 5 , 9 4 4 , 8 0 0}$ & $\mathbf{6 5 8 , 1 0 1 , 4 0 7}$ \\
\hline SEPTEMBER & $\mathbf{2 , 0 1 8 , 9 0 4 , 4 5 4}$ & $\mathbf{1 , 4 2 2 , 3 6 8 , 0 0 0}$ & $\mathbf{5 9 6 , 5 3 6 , 4 5 4}$ \\
\hline OCTOBER & $\mathbf{1 , 8 3 1 , 0 7 8 , 4 7 4}$ & $\mathbf{1 , 4 4 3 , 7 7 4 , 9 0 0}$ & $\mathbf{3 8 7 , 3 0 3 , 5 7 4}$ \\
\hline NOVEMBER & $\mathbf{1 , 9 5 4 , 9 2 8 , 6 9 1}$ & $\mathbf{1 , 5 5 3 , 0 3 9 , 1 0 0}$ & $\mathbf{4 0 1 , 8 8 9 , 5 9 1}$ \\
\hline DECEMBER & $\mathbf{1 , 9 8 8 , 1 2 7 , 3 3 4}$ & $\mathbf{1 , 5 6 8 , 3 4 0 , 6 0 0}$ & $\mathbf{4 1 9 , 7 8 6 , 7 3 4}$ \\
\hline \multicolumn{1}{|c|}{ TOTAL } & $\mathbf{2 1 , 5 8 4 , 5 9 8 , 6 4 1}$ & $\mathbf{1 5 , 5 1 9 , 6 9 3 , 0 0 0}$ & $\mathbf{6 , 0 6 4 , 9 0 5 , 6 4 1}$ \\
\hline
\end{tabular}

\section{RS UMMI}

COMPARISON OF HOSPITAL RATES WITH INA-CBG'S NHI RATES

INPATIENTS IN 2017

\begin{tabular}{|l|r|r|r|}
\hline $\begin{array}{c}\text { MONTH } \\
\text { OF SER ICE }\end{array}$ & $\begin{array}{c}\text { NET NHI } \\
\text { (HOSPITAL RATES) }\end{array}$ & $\begin{array}{c}\text { NET NHI } \\
\text { (INA-CBG'S RATES) }\end{array}$ & \multicolumn{1}{c|}{ DIFFERENCE } \\
\hline JANUARY & $\mathbf{2 , 5 9 3 , 4 3 0 , 6 2 5}$ & $\mathbf{1 , 7 7 5 , 5 5 3 , 1 0 0}$ & $\mathbf{8 1 7 , 8 7 7 , 5 2 5}$ \\
\hline FEBRUARY & $\mathbf{2 , 5 7 6 , 8 8 6 , 2 5 1}$ & $\mathbf{1 , 7 3 8 , 2 8 1 , 3 0 0}$ & $\mathbf{8 3 8 , 6 0 4 , 9 5 1}$ \\
\hline MARCH & $\mathbf{3 , 0 7 1 , 7 0 1 , 6 6 8}$ & $\mathbf{1 , 9 7 6 , 9 5 2 , 0 0 0}$ & $\mathbf{1 , 0 9 4 , 7 4 9 , 6 6 8}$ \\
\hline APRIL & $\mathbf{3 , 0 3 8 , 1 8 7 , 0 9 1}$ & $\mathbf{1 , 9 0 7 , 7 3 9 , 3 0 0}$ & $\mathbf{1 , 1 3 0 , 4 4 7 , 7 9 1}$ \\
\hline MAY & $\mathbf{3 , 3 5 4 , 7 1 6 , 1 3 6}$ & $\mathbf{2 , 2 2 2 , 0 5 7 , 3 0 0}$ & $\mathbf{1 , 1 3 2 , 6 5 8 , 8 3 6}$ \\
\hline JUNE & $\mathbf{2 , 6 3 0 , 5 2 3 , 5 8 0}$ & $\mathbf{1 , 7 6 6 , 4 4 6 , 5 0 0}$ & $\mathbf{8 6 4 , 0 7 7 , 0 8 0}$ \\
\hline JULY & $\mathbf{3 , 8 0 1 , 4 0 5 , 4 9 4}$ & $\mathbf{2 , 3 4 5 , 5 5 3 , 6 0 0}$ & $\mathbf{1 , 4 5 5 , 8 5 1 , 8 9 4}$ \\
\hline AUGUST & $\mathbf{4 , 2 2 8 , 4 4 5 , 5 7 2}$ & $\mathbf{2 , 4 2 8 , 5 4 5 , 1 0 0}$ & $\mathbf{1 , 7 9 9 , 9 0 0 , 4 7 2}$ \\
\hline SEPTEMBER & $\mathbf{3 , 8 1 7 , 0 9 5 , 2 1 8}$ & $\mathbf{2 , 1 0 1 , 7 0 5 , 9 0 0}$ & $\mathbf{1 , 7 1 5 , 3 8 9 , 3 1 8}$ \\
\hline OCTOBER & $\mathbf{3 , 9 5 8 , 1 0 0 , 4 3 4}$ & $\mathbf{2 , 3 3 0 , 6 0 0 , 9 0 0}$ & $\mathbf{1 , 6 2 7 , 4 9 9 , 5 3 4}$ \\
\hline NOVEMBER & $\mathbf{4 , 1 8 7 , 4 9 3 , 8 4 2}$ & $\mathbf{2 , 3 4 3 , 6 5 2 , 6 0 0}$ & $\mathbf{1 , 8 4 3 , 8 4 1 , 2 4 2}$ \\
\hline DECEMBER & $\mathbf{4 , 8 3 8 , 5 3 5 , 3 3 0}$ & $\mathbf{2 , 8 6 5 , 7 4 7 , 3 0 0}$ & $\mathbf{1 , 9 7 2 , 7 8 8 , 0 3 0}$ \\
\hline \multicolumn{1}{|c|}{ TOTAL } & $\mathbf{4 2 , 0 9 6 , 5 2 1 , 2 4 1}$ & $\mathbf{2 5 , 8 0 2 , 8 3 4 , 9 0 0}$ & $\mathbf{1 6 , 2 9 3 , 6 8 6 , 3 4 1}$ \\
\hline
\end{tabular}

\section{RS UMMI}

COMPARISON OF HOSPITAL RATES WITH INA-CBG'S NHI RATES

INPATIENTS IN 2018

\begin{tabular}{|l|r|r|r|}
\hline $\begin{array}{c}\text { MONTH } \\
\text { OF SERVICE }\end{array}$ & $\begin{array}{c}\text { NET NHI } \\
\text { (HOSPITAL RATES) }\end{array}$ & $\begin{array}{c}\text { NET NHI } \\
\text { (INA-CBG'S RATES) }\end{array}$ & \multicolumn{1}{c|}{ DIFFERENCE } \\
\hline JANUARY & $\mathbf{5 , 5 9 3 , 0 8 5 , 9 7 4}$ & $\mathbf{3 , 3 6 4 , 3 8 1 , 3 0 0}$ & $\mathbf{2 , 2 2 8 , 7 0 4 , 6 7 4}$ \\
\hline FEBRUARY & $\mathbf{3 , 9 2 1 , 4 3 6 , 7 2 5}$ & $\mathbf{2 , 3 7 9 , 4 9 1 , 5 0 0}$ & $\mathbf{1 , 5 4 1 , 9 4 5 , 2 2 5}$ \\
\hline MARCH & $\mathbf{4 , 0 6 6 , 2 8 1 , 9 2 0}$ & $\mathbf{2 , 7 1 7 , 8 5 3 , 6 0 0}$ & $\mathbf{1 , 3 4 8 , 4 2 8 , 3 2 0}$ \\
\hline APRIL & $\mathbf{3 , 6 8 8 , 1 7 4 , 5 9 2}$ & $\mathbf{2 , 7 1 7 , 4 2 2 , 5 0 0}$ & $\mathbf{9 7 0 , 7 5 2 , 0 9 2}$ \\
\hline MAY & $\mathbf{3 , 7 4 5 , 9 2 4 , 3 0 9}$ & $\mathbf{2 , 7 9 7 , 7 8 3 , 2 0 0}$ & $\mathbf{9 4 8 , 1 4 1 , 1 0 9}$ \\
\hline JUNE & $\mathbf{3 , 5 2 0 , 7 1 0 , 3 8 6}$ & $\mathbf{2 , 6 0 8 , 9 9 4 , 1 0 0}$ & $\mathbf{9 1 1 , 7 1 6 , 2 8 6}$ \\
\hline JULY & $\mathbf{4 , 2 3 3 , 8 9 2 , 3 7 8}$ & $\mathbf{3 , 0 4 5 , 3 6 9 , 1 0 0}$ & $\mathbf{1 , 1 8 8 , 5 2 3 , 2 7 8}$ \\
\hline AUGUST & $\mathbf{4 , 3 1 4 , 3 9 6 , 5 1 0}$ & $\mathbf{2 , 8 7 7 , 1 7 9 , 7 0 0}$ & $\mathbf{1 , 4 3 7 , 2 1 6 , 8 1 0}$ \\
\hline SEPTEMBER & $\mathbf{4 , 0 6 3 , 5 5 8 , 2 9 0}$ & $\mathbf{2 , 8 4 6 , 3 0 0 , 1 0 0}$ & $\mathbf{1 , 2 1 7 , 2 5 8 , 1 9 0}$ \\
\hline OCTOBER & $\mathbf{4 , 6 6 2 , 5 3 9 , 3 1 5}$ & $\mathbf{3 , 1 1 4 , 2 1 3 , 3 0 0}$ & $\mathbf{1 , 5 4 8 , 3 2 6 , 0 1 5}$ \\
\hline NOVEMBER & $\mathbf{4 , 3 2 6 , 1 5 4 , 9 5 3}$ & $\mathbf{2 , 8 0 8 , 2 1 6 , 3 0 0}$ & $\mathbf{1 , 5 1 7 , 9 3 8 , 6 5 3}$ \\
\hline DECEMBER & $\mathbf{4 , 0 1 0 , 5 3 3 , 5 2 7}$ & $\mathbf{2 , 8 4 4 , 5 6 0 , 3 0 0}$ & $\mathbf{1 , 1 6 5 , 9 7 3 , 2 2 7}$ \\
\hline \multicolumn{1}{|c|}{ TOTAL } & $\mathbf{5 0 , 1 4 6 , 6 8 8 , 8 7 9}$ & $\mathbf{3 4 , 1 2 1 , 7 6 5 , 0 0 0}$ & $\mathbf{1 6 , 0 2 4 , 9 2 3 , 8 7 9}$ \\
\hline
\end{tabular}


Muhammad Zafrullah \& Hadri Mulya., Sch J Econ Bus Manag, Apr, 2021; 8(4): 110-135

For National Health Insurance patients for inpatient services, the difference in National Health Insurance recorded is in 2016 amounting to $\mathrm{Rp}$. $6,064,905,641$, - in 2017 amounting to $\mathrm{Rp}$. $16,293,686,341$, and in 2018 amounting to $\mathrm{Rp}$. $16,024,923,879$.

\subsection{Analysis of Load}

Ummi Hospital in reporting expenses uses the expense recognition method on an accrual basis. In addition, the company recognizes expenses based on the nature of the expenses which places the items of expenses based on the place where the expenses are used. However, due to the difference between the general hospital rates and the INA-CBG's National Health Insurance rates and the feedback period from National Health Insurance, it results in expenses being recorded on a cash basis, which of course is not in accordance with the matching cost concept.

\begin{tabular}{|c|c|c|c|}
\hline \multicolumn{4}{|c|}{$\begin{array}{l}\text { RS UMMI } \\
\text { DIFFERENCE BETWEEN NATIONAL HEALTH INSURANCE }\end{array}$} \\
\hline MONTH & 2016 & 2017 & 2018 \\
\hline January & $(340,892,745)$ & $(995,280,119)$ & $30,492,240$ \\
\hline \begin{tabular}{|l} 
February \\
\end{tabular} & $(277,631,674)$ & $(623,589,422)$ & $(5,130,407)$ \\
\hline March & $(384,268,146)$ & $(655,891,348)$ & $(4,038,896,202)$ \\
\hline April & $(564,560,897)$ & $(1,069,592,651)$ & $(1,903,097,819)$ \\
\hline May & $(\mathbf{7 8 8 , 7 9 5 , 7 4 8 )}$ & $(1,352,150,497)$ & $(638,141,714)$ \\
\hline June & $(832,886,055)$ & $(1,138,093,655)$ & $(445,322,702)$ \\
\hline July & $(769,103,673)$ & $(1,125,871,057)$ & $(1,265,958,912)$ \\
\hline August & $(816,285,433)$ & $(1,416,461,403)$ & $(1,376,066,162)$ \\
\hline September & $(\mathbf{7 7 2 , 8 7 3 , 0 7 3 )}$ & $(464,104,015)$ & $(1,273,306,343)$ \\
\hline October & $(685,595,924)$ & $(2,008,015,636)$ & $(1,484,243,957)$ \\
\hline November & $(542,560,435)$ & $(4,609,375,370)$ & $(1,142,951,407)$ \\
\hline December & $(184,943,397)$ & $(4,093,711,301)$ & $\begin{array}{l}(1,879,981,782) \\
\end{array}$ \\
\hline Total & $(6,960,397,200)$ & $(19,552,136,474)$ & $(15,422,605,167)$ \\
\hline
\end{tabular}

In 2016, the difference between the National Health Insurance was recorded at Rp. 6,960,397,200, or $12.16 \%$ of the value of income, in 2017 the value of the difference between the National Health Insurance was Rp. $19,552,136,474$, - or $23.55 \%$ of the revenue value, and in 2018 the difference National Health
Insurance in the amount of Rp. 15,422,605,167, - or $15.90 \%$ of the total income. The correction in the National Health Insurance Difference account for the recognition of its incorrect income period was caused by a feedback period or feedback on hospital claims imposed by National Health Insurance. 
RS UMMI

DETAILS OF CHARGES TO THE NHI DIFFERENCE ACCOUNT - 2016

\begin{tabular}{|c|c|c|c|}
\hline $\begin{array}{c}\text { MONTH AND YEAR } \\
\text { LOADING }\end{array}$ & VALUE & $\begin{array}{c}\text { MONTH AND YEAR } \\
\text { TRANSACTION }\end{array}$ & VALUE \\
\hline \multirow[t]{4}{*}{ January 2016} & $340,892,745$ & September 2015 & (1,440,062) \\
\hline & & October 2015 & $11,962,641$ \\
\hline & & November 2015 & $9,697,516$ \\
\hline & & December 2015 & $320,672,650$ \\
\hline \multirow[t]{4}{*}{ February 2016} & $277,631,674$ & October 2015 & $(7,549,108)$ \\
\hline & & November 2015 & $443,827,970$ \\
\hline & & December 2015 & $391,735,024$ \\
\hline & & January 2016 & $(550,382,212)$ \\
\hline \multirow[t]{4}{*}{ March 2016} & $384,268,146$ & \begin{tabular}{|l|} 
November 2015 \\
\end{tabular} & $24,038,023$ \\
\hline & & December 2015 & $6,547,062$ \\
\hline & & January 2016 & $24,609,512$ \\
\hline & & February 2016 & $329,073,549$ \\
\hline \multirow[t]{6}{*}{ April 2016} & $564,560,897$ & October 2015 & $(5,840,032)$ \\
\hline & & November 2015 & $(12,005,195)$ \\
\hline & & December 2015 & $(1,118,956)$ \\
\hline & & January 2016 & $11,467,603$ \\
\hline & & February 2016 & $90,364,787$ \\
\hline & & March 2016 & $481,692,690$ \\
\hline \multirow[t]{3}{*}{ May 2016} & $788,795,748$ & February 2016 & $23,758,338$ \\
\hline & & March 2016 & $(9,701,619)$ \\
\hline & & April 2016 & $774,739,029$ \\
\hline \multirow[t]{8}{*}{ June 2016} & $832,886,055$ & October 2015 & $(10,118,637)$ \\
\hline & & November 2015 & $(4,374,764)$ \\
\hline & & December 2015 & 969,150 \\
\hline & & January 2016 & $13,096,787$ \\
\hline & & February 2016 & $(6,113,184)$ \\
\hline & & March 2016 & $37,210,967$ \\
\hline & & April 2016 & $(43,137,423)$ \\
\hline & & May 2016 & $845,353,159$ \\
\hline
\end{tabular}


Muhammad Zafrullah \& Hadri Mulya., Sch J Econ Bus Manag, Apr, 2021; 8(4): 110-135

\begin{tabular}{|c|c|c|c|}
\hline July 2016 & $769,103,673$ & March 2016 & $23,646,187$ \\
\hline & & April 2016 & $6,449,788$ \\
\hline & & May 2016 & $739,007,698$ \\
\hline \multirow[t]{7}{*}{ August 2016} & $816,285,433$ & December 2015 & $21,573,811$ \\
\hline & & January 2016 & $1,673,557$ \\
\hline & & February 2016 & $16,575,450$ \\
\hline & & March 2016 & $(1,539,660)$ \\
\hline & & April 2016 & $209,385,162$ \\
\hline & & May 2016 & $210,777,126$ \\
\hline & & June 2016 & $357,839,987$ \\
\hline \multirow[t]{5}{*}{ September 2016} & 772,873,073 & April 2016 & $34,568,220$ \\
\hline & & May 2016 & $3,446,244$ \\
\hline & & June 2016 & $72,344,813$ \\
\hline & & July 2016 & $216,198,819$ \\
\hline & & August 2016 & $446,314,977$ \\
\hline \multirow[t]{6}{*}{ October 2016} & 685,595,924 & April 2016 & $41,821,700$ \\
\hline & & May 2016 & $31,954,032$ \\
\hline & & June 2016 & $73,013,537$ \\
\hline & & July 2016 & $492,670,350$ \\
\hline & & August 2016 & $437,234,973$ \\
\hline & & September 2016 & $(391,098,668)$ \\
\hline \multirow[t]{4}{*}{ November 2016} & $542,560,435$ & December 2015 & $3,010,576$ \\
\hline & & July 2016 & $13,607,868$ \\
\hline & & August 2016 & $11,255,430$ \\
\hline & & September 2016 & 514,686,561 \\
\hline \multirow[t]{11}{*}{ December 2016} & 184,943,397 & January 2016 & 23,269 \\
\hline & & February 2016 & $(2,911,387)$ \\
\hline & & March 2016 & $(3,968,648)$ \\
\hline & & April 2016 & $4,417,596$ \\
\hline & & May 2016 & $2,725,583$ \\
\hline & & June 2016 & $(4,628,854)$ \\
\hline & & July 2016 & $\mathbf{2 7 , 0 2 8 , 5 6 7}$ \\
\hline & & August 2016 & $(2,325,858)$ \\
\hline & & September 2016 & $99,450,076$ \\
\hline & & October 2016 & $419,504,709$ \\
\hline & & November 2016 & $(354,371,656)$ \\
\hline Total & $\mathbf{6 , 9 6 0 , 3 9 7 , 2 0 0}$ & & $\overline{6,960,397,200}$ \\
\hline
\end{tabular}

From the analysis, the correction to 2015 income recorded in the National Health Insurance
Difference account and charged in 2016 amounted to Rp. 1,191,587,669, - . 
RS UMMI

DETAILS OF CHARGES TO THE NHI DIFFERENCE ACCOUNT - 2017

\begin{tabular}{|c|c|c|c|}
\hline $\begin{array}{c}\text { MONTH AND YEAR } \\
\text { LOADING } \\
\end{array}$ & VALUE & $\begin{array}{c}\text { MONTH AND YEAR } \\
\text { TRANSACTION } \\
\end{array}$ & VALUE \\
\hline \multirow[t]{5}{*}{ January 2017} & $995,280,119$ & August 2016 & $15,994,457$ \\
\hline & & September 2016 & $31,935,405$ \\
\hline & & October 2016 & $1,579,123$ \\
\hline & & November 2016 & $450,556,793$ \\
\hline & & December 2016 & $495,214,341$ \\
\hline \multirow[t]{4}{*}{ February 2017} & $623,589,422$ & October 2016 & $7,329,420$ \\
\hline & & November 2016 & $6,282,473$ \\
\hline & & December 2016 & $10,008,826$ \\
\hline & & January 2017 & $599,968,703$ \\
\hline \multirow{3}{*}{ March 2017} & $655,891,348$ & October 2016 & $37,975,545$ \\
\hline & & January 2017 & $617,786,329$ \\
\hline & & February 2017 & 129,474 \\
\hline \multirow{3}{*}{ April 2017} & $1,069.592 .651$ & \begin{tabular}{|l|} 
January 2017 \\
\end{tabular} & $107,046,748$ \\
\hline & & \begin{tabular}{|l|} 
February 2017 \\
\end{tabular} & $65,733,148$ \\
\hline & & March 2017 & $896,812,755$ \\
\hline \multirow[t]{9}{*}{ May 2017} & $1,352,150,497$ & August 2016 & $(530,181)$ \\
\hline & & September 2016 & $(3,350,849)$ \\
\hline & & October 2016 & $16,025,397$ \\
\hline & & November 2016 & $(7,393,796)$ \\
\hline & & December 2016 & $17,792,168$ \\
\hline & & January 2017 & $23,527,392$ \\
\hline & & February 2017 & $22,581,553$ \\
\hline & & March 2017 & $610,492,568$ \\
\hline & & April 2017 & $673,006,245$ \\
\hline \multirow{11}{*}{ June 2017} & $1,138,093,655$ & January 2017 & $(201,852)$ \\
\hline & & February 2016 & 108,805 \\
\hline & & Maret 2016 & 572,111 \\
\hline & & April 2016 & 31,480 \\
\hline & & July 2016 & $(43,230)$ \\
\hline & & \begin{tabular}{|l|} 
August 2016 \\
\end{tabular} & $(148,220)$ \\
\hline & & September 2016 & $(3,058,897)$ \\
\hline & & February 2017 & $11,730,344$ \\
\hline & & March 2017 & $30,017,186$ \\
\hline & & April 2017 & $79,813,156$ \\
\hline & & May 2017 & $1,019,272,772$ \\
\hline
\end{tabular}


Muhammad Zafrullah \& Hadri Mulya., Sch J Econ Bus Manag, Apr, 2021; 8(4): 110-135

\begin{tabular}{|c|c|c|c|}
\hline July 2017 & $\mathbf{1 , 1 2 5 , 8 7 1 , 0 5 7}$ & January 2017 & $5,686,102$ \\
\hline & & February 2017 & $(2,082,546)$ \\
\hline & & April 2017 & $45,121,827$ \\
\hline & & May 2017 & $215,461,286$ \\
\hline & & June 2017 & $861,684,388$ \\
\hline \multirow[t]{6}{*}{ August 2017} & $1,416,461,403$ & February 2017 & $(5,447,264)$ \\
\hline & & March 2017 & $13,300,256$ \\
\hline & & April 2017 & 110,886 \\
\hline & & \begin{tabular}{|l|} 
May 2017 \\
\end{tabular} & $9,977,205$ \\
\hline & & June 2017 & $65,625,179$ \\
\hline & & \begin{tabular}{|l|} 
July 2017 \\
\end{tabular} & $1,332,895,141$ \\
\hline \multirow[t]{3}{*}{ September 2017} & $464,104,015$ & February 2017 & $5,292,119$ \\
\hline & & July 2017 & $145,317,979$ \\
\hline & & August 2017 & $313,493,917$ \\
\hline \multirow[t]{2}{*}{ October 2017} & $2,008,015,636$ & August 2017 & $1,476,835,881$ \\
\hline & & September 2017 & $531,179,755$ \\
\hline \multirow[t]{6}{*}{ November 2017} & $4,609,375,370$ & June 2017 & $45,736,273$ \\
\hline & & July 2017 & $211,382,355$ \\
\hline & & August 2017 & $237,223,581$ \\
\hline & & September 2017 & $1,379,697,378$ \\
\hline & & October 2017 & $1,938,869,950$ \\
\hline & & November 2017 & $796,465,833$ \\
\hline \multirow[t]{8}{*}{ December 2017} & $4,093,711,301$ & February 2017 & 747,964,683 \\
\hline & & April 2017 & $621,555,060$ \\
\hline & & May 2017 & $378,444,940$ \\
\hline & & July 2017 & $316,638,870$ \\
\hline & & August 2017 & $10,734,874$ \\
\hline & & September 2017 & $135,685,578$ \\
\hline & & October 2017 & 435,594,471 \\
\hline & & \begin{tabular}{|l|} 
November 2017 \\
\end{tabular} & $1,447,092,825$ \\
\hline Total & (19,552,136,474 & & $1 \overline{19,552,136,474}$ \\
\hline
\end{tabular}

From the analysis, the correction of 2016 income recorded in the National Health Insurance
Difference account and charged in 2017 amounted to Rp. 1,176,679,319, - 
RS UMMI

DETAILS OF CHARGES TO THE NHI DIFFERENCE ACCOUNT - 2018

\begin{tabular}{|c|c|c|c|}
\hline $\begin{array}{c}\text { MONTH AND YEAR } \\
\text { LOADING }\end{array}$ & VALUE & $\begin{array}{c}\text { MONTH AND YEAR } \\
\text { TRANSACTION }\end{array}$ & VALUE \\
\hline January 2018 & (30,492,240) & December 2017 & $(30,492,240)$ \\
\hline February 2018 & 5,130,407 & December 2017 & $5,130,407$ \\
\hline March 2018 & $4,038,896,202$ & January 2018 & $4,038,896,202$ \\
\hline April 2018 & $\mathbf{1 , 9 0 3 , 0 9 7 , 8 1 9}$ & March 2018 & $\mathbf{1 , 9 0 3 , 0 9 7 , 8 1 9}$ \\
\hline \multirow[t]{2}{*}{ May 2018} & $638,141,714$ & March 2018 & $9,547,617$ \\
\hline & & April 2018 & $628,594,097$ \\
\hline \multirow[t]{2}{*}{ June 2018} & $445,322,702$ & February 2018 & $179,693,908$ \\
\hline & & March 2018 & $265,628,794$ \\
\hline \multirow[t]{3}{*}{ July 2018} & $1,265,958,912$ & April 2018 & $115,974,230$ \\
\hline & & May 2018 & $533,970,402$ \\
\hline & & \begin{tabular}{|l|} 
June 2018 \\
\end{tabular} & $616,014,280$ \\
\hline \multirow[t]{5}{*}{ August 2018} & $1,376,066,162$ & January 2018 & $56,552,526$ \\
\hline & & February 2018 & $216,191,556$ \\
\hline & & April 2018 & $1,204,473$ \\
\hline & & May 2018 & $275,923,255$ \\
\hline & & July 2018 & $826,194,352$ \\
\hline \multirow[t]{3}{*}{ September 2018} & $1,273,306,343$ & January 2018 & $149,920,285$ \\
\hline & & \begin{tabular}{|l|} 
June 2018 \\
\end{tabular} & $166,759,020$ \\
\hline & & August 2018 & $956,627,038$ \\
\hline \multirow[t]{5}{*}{ October 2018} & $1,484,243,957$ & February 2018 & $47,045,366$ \\
\hline & & April 2018 & $38,582,647$ \\
\hline & & July 2018 & $297,260,125$ \\
\hline & & August 2018 & $377,369,180$ \\
\hline & & September 2018 & $723,986,639$ \\
\hline November 2018 & $1,142,951,407$ & October 2018 & $1,142,951,407$ \\
\hline \multirow[t]{2}{*}{ December 2018} & $1,879,981,782$ & October 2018 & $460,506,921$ \\
\hline & & November 2018 & $1,419,474,861$ \\
\hline Total & $15,422,605,167$ & & $15,422,605,167$ \\
\hline
\end{tabular}

From the analysis, the correction to 2017 income recorded in the National Health Insurance
Difference account and charged in 2018 is (Rp. 25,361,833). 
Muhammad Zafrullah \& Hadri Mulya., Sch J Econ Bus Manag, Apr, 2021; 8(4): 110-135

\begin{tabular}{|c|c|c|c|c|c|c|c|c|c|}
\hline \multicolumn{10}{|c|}{$\begin{array}{l}\text { RS UMMI } \\
\text { PAYMENT O } \\
2015 \\
\text { DOCTORS }\end{array}$} \\
\hline & MONTH & $\begin{array}{l}\text { Medical Services } \\
\text { NHI Outpatient }\end{array}$ & $\begin{array}{l}\text { Medical Services } \\
\text { NHI Inpatient }\end{array}$ & $\begin{array}{c}\text { GUARANTEE FEE } \\
\text { (ABOVE GF) }\end{array}$ & TOTAL & TAX ART.21 & $\begin{array}{l}\text { ACCOUNT } \\
\text { PAYABLE }\end{array}$ & PAID & $\begin{array}{l}\text { PAYMENT } \\
\text { DATE }\end{array}$ \\
\hline 1 & Aug-15 & & $2,845,500$ & & $2,845,500$ & 213,413 & & $2,632,088$ & 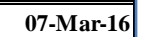 \\
\hline 2 & Sep-15 & $70,984,880$ & $212,373,183$ & $(19,997,874)$ & $263,360,189$ & $19,221,831$ & & $244,138,357$ & 08-Jan-16 \\
\hline 3 & Oct-15 & $120,234,436$ & $235,877,625$ & $(5,243,890)$ & $350,868,171$ & $8,771,704$ & $20,206,973$ & $321,889,494$ & 02-Feb-16 \\
\hline 4 & Nov-15 & $132,115,300$ & $350,829,344$ & $(18,518,200)$ & $464,426,444$ & $18,202,342$ & $15,227,643$ & $430,996,459$ & 08-Apr-16 \\
\hline \multirow{2}{*}{5} & \multirow{2}{*}{ Dec-15 } & $28,630,000$ & $35,321,415$ & - & $63,951,415$ & $1,598,785$ & $2,083,575$ & $60,269,055$ & 08-Apr-16 \\
\hline & & $104,472,076$ & $268,283,650$ & $(25,246,469)$ & $347,509,257$ & $21,366,689$ & $17,409,109$ & $308,733,460$ & 23-May-16 \\
\hline \multicolumn{2}{|c|}{ TOTAL } & $456,436,692$ & $1,105,530,716$ & $(69,006,433)$ & $\begin{array}{l}1,492,960,975 \\
\end{array}$ & $699,374,764$ & $\begin{array}{r}54,927,300 \\
\end{array}$ & $1,368,658,912$ & \\
\hline \multicolumn{10}{|c|}{ ANESTHESIOLOGIST \& ANESTHESIA ASSISTANT (OUTSIDE NURSE) } \\
\hline \multicolumn{2}{|c|}{ NQMONTH } & \begin{tabular}{c|} 
Medical Services \\
NHI Outpatient
\end{tabular} & $\begin{array}{l}\text { Medical Services } \\
\text { NHI Inpatient }\end{array}$ & $\begin{array}{c}\text { GUARANTEE FEE } \\
\text { (ABOVE GF) }\end{array}$ & TOTAL & TAX ART.21 & $\begin{array}{l}\text { ACCOUNT } \\
\text { PAYABLE }\end{array}$ & PAID & $\begin{array}{l}\text { PAYMENT } \\
\text { DATE }\end{array}$ \\
\hline 1 & Sep-15 & - & $8,361,550$ & - & $8,361,550$ & 222,264 & 625 & $8,138,661$ & 05-Jan-16 \\
\hline 2 & Oct-15 & - & $7,777,700$ & - & $\mathbf{7 , 7 7 7 , 7 0 0}$ & 211,686 & - & $7,566,014$ & 03-Feb-16 \\
\hline 3 & Nov-15 & - & $11,147,550$ & - & $11,147,550$ & 304,595 & - & $10,842,955$ & 14-Mar-16 \\
\hline & Dec-15 & - & $6,806,000$ & - & $6,806,000$ & 186,055 & - & $6,619,945$ & 09-May-16 \\
\hline & TOTAL & - & $34,092,800$ & - & $34,092,800$ & 924,600 & 625 & $33,167,575$ & \\
\hline
\end{tabular}

\section{RS UMMI}

PAYMENT OF MEDICAL SERVICES (DOCTORS AND OTHER MEDICAL SERVICES) FOR NATIONAL HEALTH INSURANCE PATIENTS 2016

\begin{tabular}{|c|c|c|c|c|c|c|c|c|c|}
\hline \\
\hline NC & MONTH & $\begin{array}{l}\text { Medical Services } \\
\text { NHI Outpatient }\end{array}$ & $\begin{array}{l}\text { Medical Services } \\
\text { NHI Inpatient }\end{array}$ & $\begin{array}{l}\text { GUARANTEE FEE } \\
\text { (ABOVE GF) }\end{array}$ & TOTAL & TAX ART.21 & $\begin{array}{l}\text { ACCOUNT } \\
\text { PAYABLE }\end{array}$ & PAID & $\begin{array}{l}\text { PAYMENT } \\
\text { DATE }\end{array}$ \\
\hline 1 & Jan-16 & $164,793,280$ & $271,493,035$ & - & $436,286,315$ & $25,310,201$ & 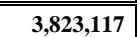 & $407,152,997$ & 06-Jun-16 \\
\hline \multirow{2}{*}{2} & \multirow{2}{*}{ Feb-16 } & $61,635,000$ & $175,550,795$ & - & $237,185,795$ & $17,427,572$ & $3,857,685$ & $215,900,537$ & 29-Jun-16 \\
\hline & & 77,985,488 & $107,484,262$ & - & $185,469,750$ & $10,596,703$ & $22,754,297$ & $152,118,750$ & 01-Jul-16 \\
\hline \multirow{3}{*}{3} & \multirow{3}{*}{ Mar-16 } & $\begin{array}{l}6,720,000 \\
\end{array}$ & $1,765,000$ & - & $8,485,000$ & 212,125 & $\begin{array}{r}54,210 \\
\end{array}$ & $8,218,665$ & 20-Jul-16 \\
\hline & & $176,405,590$ & $230,124,167$ & - & $406,529,757$ & $31,599,975$ & $22,050,438$ & $352,879,344$ & 04-Aug-16 \\
\hline & & $2,304,750$ & $1,364,250$ & - & $3,669,000$ & 91,725 & $1,869,600$ & $1,707,675$ & 16-Sep-16 \\
\hline \multirow{2}{*}{4} & \multirow{2}{*}{ Apr-16 } & $131,692,000$ & $234,444,590$ & - & $366,136,590$ & $30,646,229$ & $28,061,267$ & $307,429,094$ & 31-Aug-16 \\
\hline & & $\mathbf{9 0 , 5 1 4 , 7 9 0}$ & \begin{tabular}{|l|}
$11,286,020$ \\
\end{tabular} & - & $101,800,810$ & \begin{tabular}{|l|}
$6,973,482$ \\
\end{tabular} & - & $94,827,329$ & 16-Sep-16 \\
\hline 5 & May-16 & $193,066,235$ & $271,095,410$ & $(25,751,260)$ & $438,410,385$ & $38,473,576$ & $2,092,126$ & $397,844,683$ & 05-Oct-16 \\
\hline 6 & \begin{tabular}{|l|} 
Jun-16 \\
\end{tabular} & $158,801,550$ & $242,064,254$ & $(26,177,800)$ & $374,688,004$ & $36,260,138$ & $1,814,780$ & $336,613,086$ & 14-Oct-16 \\
\hline 7 & Jul-16 & $127,215,825$ & $217,463,825$ & $(28,234,750)$ & $316,444,900$ & $29,891,337$ & $1,586,956$ & $284,966,607$ & 04-Nov-16 \\
\hline \multirow{2}{*}{8} & \multirow{2}{*}{ Aug-16 } & $160,662,500$ & $226,957,354$ & $(17,993,900)$ & $369,625,954$ & $\mathbf{3 5 , 6 3 8 , 3 0 7}$ & $2,666,912$ & $331,320,735$ & 27-Dec-16 \\
\hline & & $2,220,825$ & $28,055,941$ & & $100,276,766$ & \begin{tabular}{|l}
$9,634,175$ \\
\end{tabular} & & $90,642,591$ & 09-Jan-17 \\
\hline 9 & Sep-16 & $216,980,463$ & $300,588,033$ & $(20,042,500)$ & $497,525,996$ & $12,452,672$ & $3,109,074$ & $481,964,250$ & 18-Jan-17 \\
\hline \multirow{2}{*}{10} & \multirow{2}{*}{ Oct-16 } & $220,685,111$ & $263,542,473$ & $(22,460,780)$ & $461,766,804$ & $11,544,170$ & $10,000,000$ & $440,222,634$ & 31-Jan-17 \\
\hline & & $1,960,000$ & $6,007,772$ & & $7,967,772$ & $\begin{array}{l}199,194 \\
\end{array}$ & $2,379,345$ & \begin{tabular}{|l|}
$5,389,233$ \\
\end{tabular} & 01-Feb-17 \\
\hline 11 & Nov-16 & $239,057,876$ & $297,581,756$ & $(22,673,550)$ & $513,966,082$ & $24,374,372$ & $17, \mathbf{4 3 4 , 4 4 7}$ & $472,157,263$ & 21-Feb-17 \\
\hline 12 & Dec-16 & $208,035,611$ & $320,127,798$ & $(21,359,600)$ & $506,803,809$ & $28,156,089$ & $12,408,190$ & $466,239,530$ & 14-Mar-17 \\
\hline & TOTAL & $2,310,736,894$ & $3,206,996,735$ & $(184,694,140)$ & $5,333,039,489$ & $349,482,043$ & $135,962,444$ & $4,847,595,001$ & \\
\hline
\end{tabular}

ANESTHESIOLOGIST \& ANESTHESIA ASSISTANT (OUTSIDE NURSE)

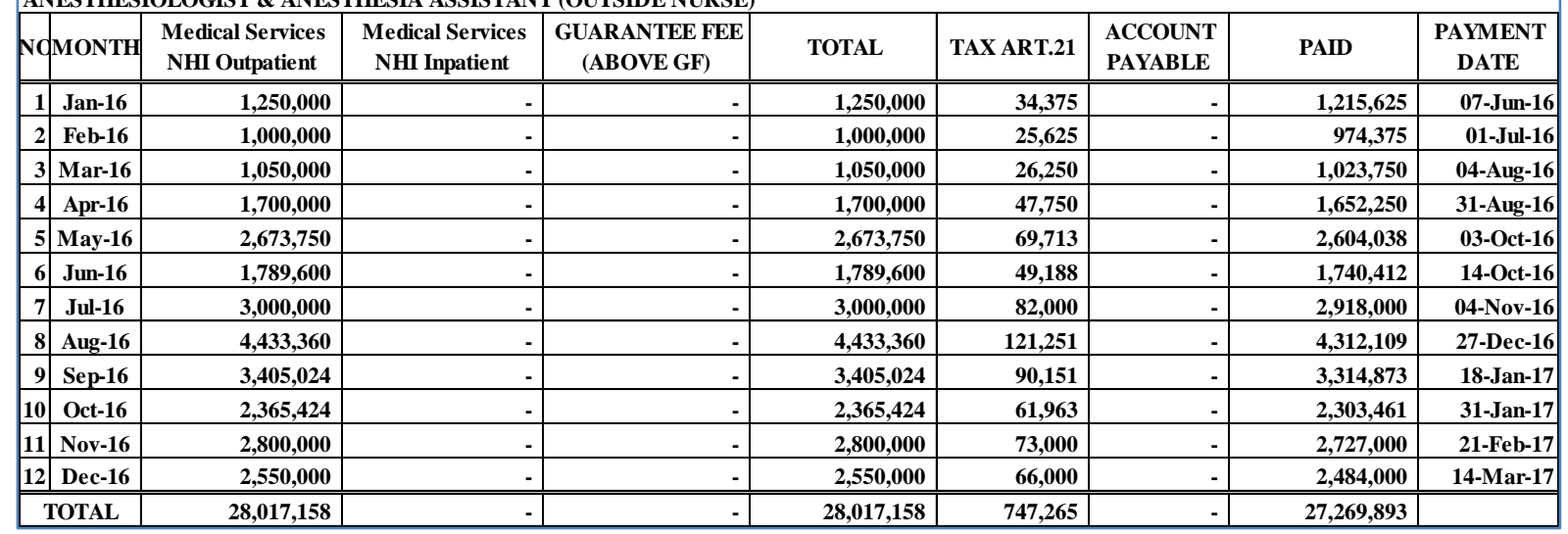




\begin{tabular}{|c|c|c|c|c|c|c|c|c|c|c|}
\hline \multicolumn{11}{|c|}{$\begin{array}{l}\text { RS UMMI } \\
\text { PAYMENT } \\
2017 \\
\text { DOCTORS }\end{array}$} \\
\hline $\mathrm{ndn}$ & MONTH & $\begin{array}{l}\text { Medical Services } \\
\text { NHI Outpatient }\end{array}$ & $\begin{array}{l}\text { Medical Services } \\
\text { NHI Inpatient }\end{array}$ & $\begin{array}{c}\text { IGD } \\
\text { Reference }\end{array}$ & $\begin{array}{l}\text { GUARANTEE FEE } \\
\text { (ABOVE GF) }\end{array}$ & TOTAL & TAX ART.21 & $\begin{array}{l}\text { ACCOUNT } \\
\text { PAYABLE }\end{array}$ & PAID & $\begin{array}{l}\text { PAYMENT } \\
\text { DATE }\end{array}$ \\
\hline \multirow{2}{*}{1} & \multirow{2}{*}{ Jan-17 } & $105,043,072$ & $121,442,358$ & - & $13,333,900$ & $213,151,530$ & $\mathbf{9 , 1 3 5 , 7 7 3}$ & $5,397,301$ & $198,618,456$ & 28-Apr-17 \\
\hline & & $112,770,000$ & $279,228,615$ & - & $(3,234,700)$ & $388,763,915$ & $27, \mathbf{8 3 2}, 353$ & - & $360,931,562$ & 04-May-17 \\
\hline 2 & Feb-17 & $230,875,798$ & 335,629,788 & - & $(48,619,283)$ & $517,886,303$ & $39,692,452$ & $3,748,000$ & 474,445,851 & 30-May-17 \\
\hline 3 & Mar-17 & $312,255,928$ & $405,579,956$ & - & $(37,228,005)$ & $680,607,879$ & $56,416,680$ & $14,799,634$ & $609,391,565$ & 05-Jun-17 \\
\hline 4 & Apr-17 & $257,649,836$ & 436,054,389 & - & $(33,047,710)$ & $660,656,515$ & $60,680,485$ & $13,186,868$ & $586,789,162$ & 27-Jul-17 \\
\hline 5 & May-17 & $314,637,015$ & $447,592,931$ & - & $(52,759,045)$ & 709,470,901 & $70,918,486$ & - & $638,552,415$ & 30-Aug-17 \\
\hline \multirow[b]{2}{*}{6} & \multirow{2}{*}{ Jun-17 } & $158,290,000$ & $230,810,782$ & - & $(51,917,550)$ & 337,183,232 & $31,650,848$ & - & 305,532,384 & 20-Sep-17 \\
\hline & & $64,369,029$ & $126,597,059$ & - & $(1,610,170)$ & $189,355,918$ & $25,258,716$ & - & $164,097,202$ & 04-Oct-17 \\
\hline \multirow[b]{3}{*}{7} & \multirow{3}{*}{ Jul-17 } & $10,360,000$ & $58,108,580$ & - & $(11,752,000)$ & $56,716,580$ & $1,417,915$ & - & $55,298,666$ & 15-Sep-17 \\
\hline & & $87,525,000$ & $40,705,500$ & - & $(547,000)$ & $127,683,500$ & $11,622,538$ & - & $116,060,963$ & 31-Oct-17 \\
\hline & & $187,094,189$ & $395,710,318$ & - & $(39,894,950)$ & $542,909,557$ & $60,626,265$ & $4,000,000$ & $478,283,292$ & 14-Nov-17 \\
\hline \multirow[b]{3}{*}{8} & \multirow{3}{*}{ Aug-17 } & $17,920,000$ & $55,920,900$ & - & - & $73,840,900$ & $4,575,140$ & $1,027,620$ & $68,238,141$ & 27-Oct-17 \\
\hline & & $7,265,000$ & $14,092,240$ & - & $(5,696,000)$ & $15,661,240$ & 391,531 & - & $15,269,709$ & 01-Nov-17 \\
\hline & & $342,619,437$ & $296,415,626$ & $6,325,000$ & $(53,766,770)$ & $591,593,293$ & $59,454,534$ & $5,000,000$ & $527,138,759$ & 06-Dec-17 \\
\hline \multirow[b]{2}{*}{9} & \multirow{2}{*}{ Sep-17 } & $14,315,000$ & $37,729,900$ & - & $(4,313,300)$ & $47,731,600$ & $3,579,870$ & $1,180,067$ & $42,971,663$ & 13-Nov-17 \\
\hline & & $300,770,840$ & $353,337,563$ & $4,025,000$ & $(49,171,770)$ & $608,961,633$ & $62,067,261$ & $6,000,000$ & $540,894,372$ & 11-Dec-17 \\
\hline \multirow{3}{*}{\multicolumn{2}{|c|}{ Oct-17 }} & $3,150,000$ & $3,730,000$ & - & $(1,500,000)$ & $5,380,000$ & 134,500 & $2,242,500$ & $3,003,000$ & 15-Dec-17 \\
\hline & & $43,235,430$ & $27,339,801$ & - & $(14,182,420)$ & $56,392,811$ & $1,409,820$ & $10,294,675$ & $44,688,316$ & 25-Jan-18 \\
\hline 10 & & $317,029,235$ & $416,752,929$ & $9,300,000$ & $(35,870,569)$ & $707,211,595$ & $21,801,822$ & $80,794,675$ & $604,615,098$ & 14-Feb-18 \\
\hline 11 & Nov-17 & $428,987,175$ & $522,618,800$ & $10,700,000$ & $(71,581,050)$ & $890,724,925$ & $42,724,263$ & $57,129,843$ & $790,870,819$ & 20-Mar-18 \\
\hline \begin{tabular}{|l|l|}
12 \\
\end{tabular} & Dec-17 & $405,412,989$ & $552,462,045$ & $10,175,000$ & $63,588,880$ & $904,461,154$ & $50,829,029$ & $73,250,433$ & $780,381,692$ & 16-Apr-18 \\
\hline & TOTAL & $3,721,574,973$ & $5,157,860,080$ & $40,525,000$ & 593,615,072 & $8,326,344,980$ & $642,220,279$ & $278,051,616$ & $7,406,073,085$ & \\
\hline \multicolumn{11}{|c|}{ ANESTHESIOLOGIST \& ANESTHESIA ASSISTANT (OUTSIDE NURSE) } \\
\hline \multicolumn{2}{|c|}{ NOMONTH } & $\begin{array}{l}\text { Medical Services } \\
\text { NHI Outpatient }\end{array}$ & $\begin{array}{c}\text { Medical Services } \\
\text { NHI Inpatient }\end{array}$ & $\begin{array}{c}\text { IGD } \\
\text { Reference }\end{array}$ & $\begin{array}{c}\text { GUARANTEE FEE } \\
\text { (ABOVE GF) }\end{array}$ & TOTAL & TAX ART.21 & $\begin{array}{l}\text { ACCOUNT } \\
\text { PAYABLE }\end{array}$ & PAID & $\begin{array}{l}\text { PAYMENT } \\
\text { DATE }\end{array}$ \\
\hline 1 & Jan-17 & $5,489,600$ & - & - & - & $5,489,600$ & 146,688 & - & $5,342,912$ & 04-May-17 \\
\hline 2 & Feb-17 & $4,200,000$ & - & - & - & $4,200,000$ & 110,250 & - & $4,089,750$ & 16-May-17 \\
\hline 3 & Mar-17 & $4,000,000$ & - & - & - & $4,000,000$ & 103,250 & - & $3,896,750$ & 05-Jun-17 \\
\hline 4 & Apr-17 & $4,200,000$ & - & - & - & $4,200,000$ & 109,250 & - & $4,090,750$ & 27-Jul-17 \\
\hline 5 & May-17 & $4,150,000$ & - & - & - & $4,150,000$ & 106,750 & - & $4,043,250$ & 30-Aug-17 \\
\hline 6 & Jun-17 & $4,200,000$ & - & - & - & $4,200,000$ & 107,500 & - & $4,092,500$ & 04-Oct-17 \\
\hline 7 & Jul-17 & $5,850,000$ & - & - & - & $5,850,000$ & 149,250 & - & $5,700,750$ & 14-Nov-17 \\
\hline 8 & Aug-17 & $3,750,000$ & - & - & - & $3,750,000$ & 99,500 & - & $3,650,500$ & 06-Dec-17 \\
\hline 9 & Sep-17 & $6,050,000$ & - & - & - & $6,050,000$ & 162,000 & - & $5,888,000$ & 08-Dec-17 \\
\hline 10 & Oct-17 & $4,750,000$ & - & - & - & $4,750,000$ & 118,750 & - & $4,631,250$ & 14-Feb-18 \\
\hline 11 & Nov-17 & $4,850,000$ & - & - & - & $4,850,000$ & 121,250 & - & $4,728,750$ & 20-Mar-18 \\
\hline & Dec-17 & $9,700,000$ & - & - & - & $9,700,000$ & 242,500 & - & $9,457,500$ & 30-Jul-18 \\
\hline & TOTAL & $61,189,600$ & - & - & - & $61,189,600$ & $1,576,938$ & - & $59,612,662$ & \\
\hline
\end{tabular}

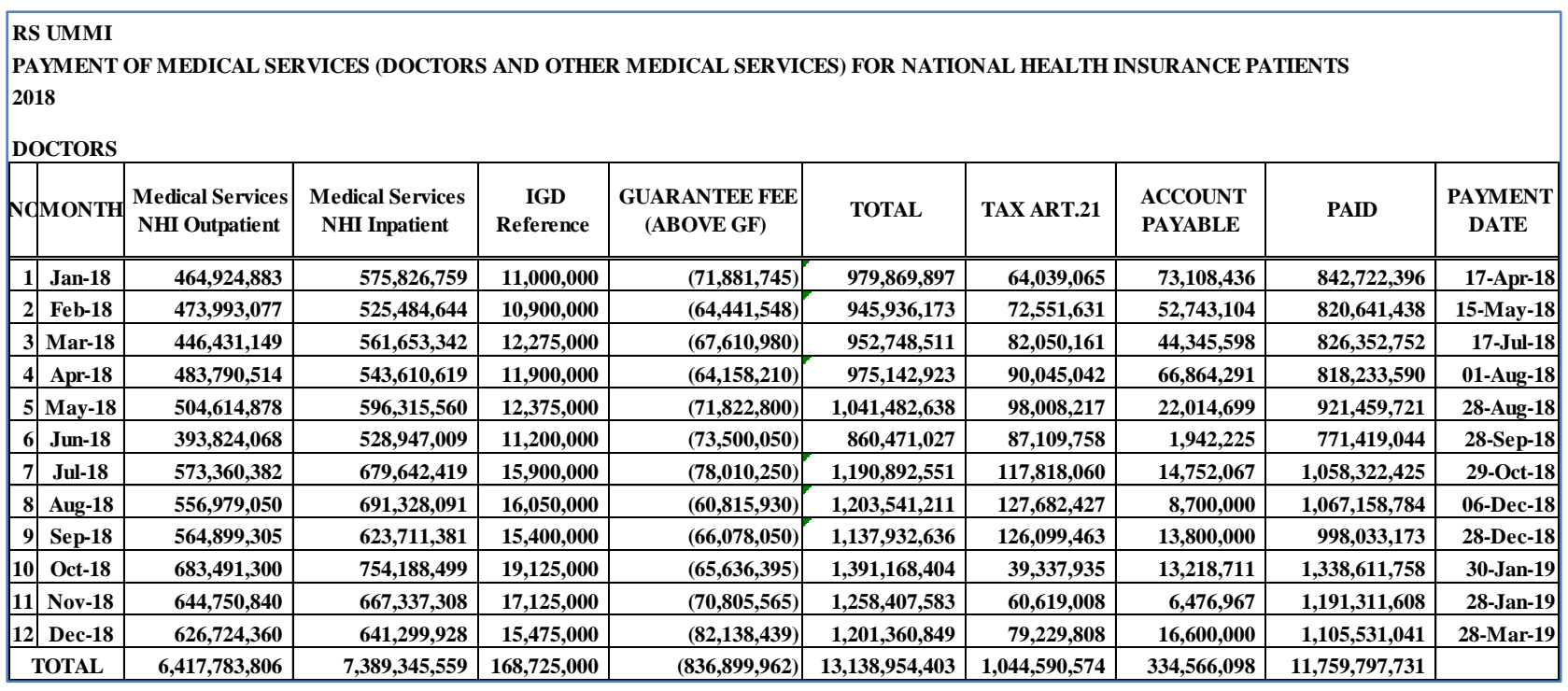


Muhammad Zafrullah \& Hadri Mulya., Sch J Econ Bus Manag, Apr, 2021; 8(4): 110-135

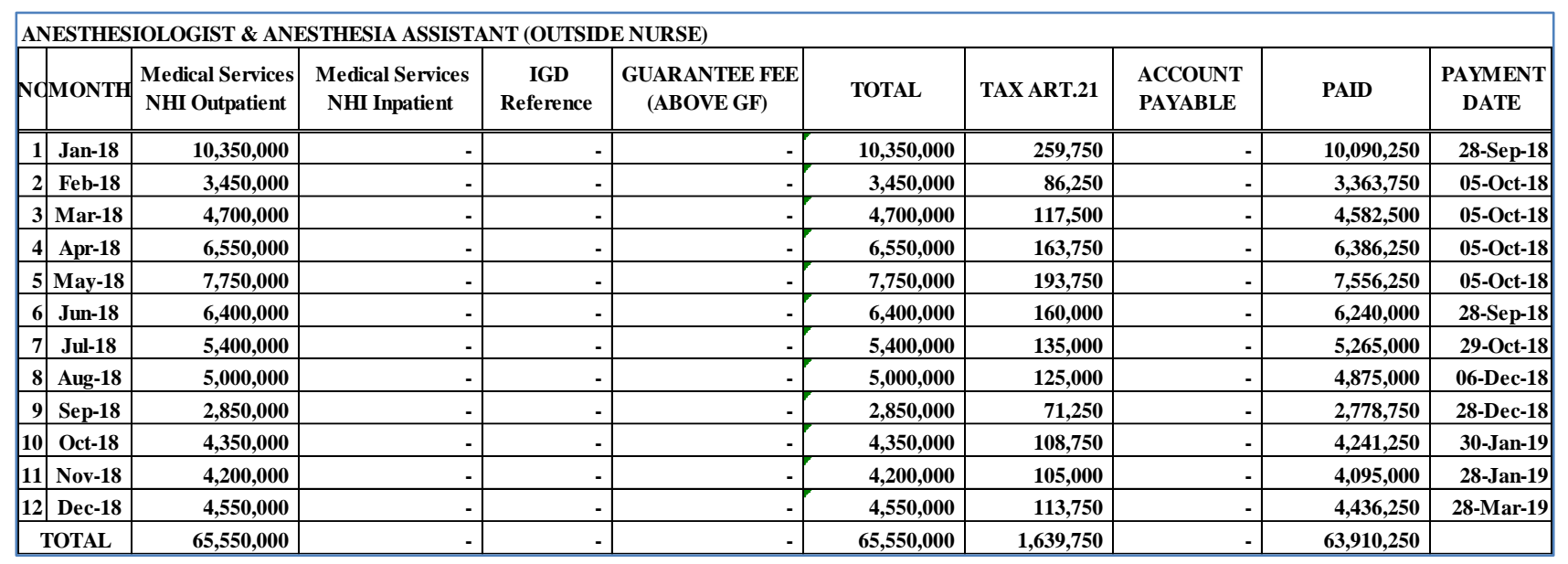

From the analysis above, it can be explained that there are charges for medical services which include payments for doctor and nurse services or medical teams that are not in accordance with the period by recording income or recorded on a cash basis:

a. In 2015 amounting to Rp. 1,401,826,487, the charges were recorded in 2016.

b. In 2016, the amount of Rp. 1,967,444,834, was recorded in 2017. c. In 2017 , the amount of Rp. 2,239,373,424, was recorded in 2018.

d. And in 2018 the amount of Rp. 3,648,226,908, was recorded in 2019.

\subsection{Analysis of Comparison of INA-CBG's National} Health Insurance Rates and Hospital Regular Rates.

The following is a comparison of the INACBG's rate value with Ummi's regular hospital rates of several medical procedures as follows:

\begin{tabular}{|c|c|c|c|c|c|c|c|c|c|c|c|c|}
\hline \multirow[b]{2}{*}{ No } & \multirow[b]{2}{*}{ ICD-10 } & \multirow[b]{2}{*}{ Description } & \multicolumn{8}{|c|}{ Hospital Rates } & \multirow[b]{2}{*}{$\begin{array}{l}\text { INA- } \\
\text { CBG's } \\
\text { Rates }\end{array}$} & \multirow[b]{2}{*}{ Defference } \\
\hline & & & Action & Consul / Visit & Nursing & $\begin{array}{c}\text { Medical } \\
\text { Support (Lab, } \\
\text { Radiology, } \\
\text { Rehabilitation) }\end{array}$ & $\begin{array}{c}\text { Room/ } \\
\text { Accomodation }\end{array}$ & $\begin{array}{c}\text { Drug/Medical } \\
\text { Devices/ } \\
\text { Consumable } \\
\text { Medical } \\
\text { Materials }\end{array}$ & $\begin{array}{l}\text { Rental } \\
\text { Tools }\end{array}$ & Total & & \\
\hline 1 & P03.4 & $\begin{array}{l}\text { Fetus and newborn affected by } \\
\text { caesarean delivery }\end{array}$ & 106,903 & 183,315 & 177,251 & 28,134 & 369,850 & 188,830 & 320,431 & $1,374,715$ & $4,121,830$ & $(2,747,116)$ \\
\hline 2 & A09.9 & $\begin{array}{l}\text { Gastroenteritis and colitis of } \\
\text { unspecified origin }\end{array}$ & 9,283 & 523,819 & 570,276 & 228,283 & 933,754 & 655,915 & 8,574 & $2,929,904$ & $1,748,098$ & $1,181,806$ \\
\hline 3 & 034.2 & $\begin{array}{l}\text { Maternal care due to uterine scar } \\
\text { from previous surgery }\end{array}$ & $2,841,475$ & 297,022 & 193,067 & 471,162 & $2,388,463$ & $1,575,566$ & 57,605 & $7,824,360$ & $5,918,270$ & $1,906,090$ \\
\hline 4 & A91 & Dengue haemorrhagic fever & 6,661 & 661,364 & 292,644 & 489,271 & $1,212,586$ & 866,580 & 39,671 & $3,568,776$ & $1,975,218$ & $1,593,559$ \\
\hline 5 & J18.0 & Bronchopneumonia, unspecified & 27,492 & 637,107 & 222,827 & 404,207 & $1,195,319$ & $1,149,753$ & 237,309 & $3,874,014$ & $4,469,221$ & $(595,207)$ \\
\hline 6 & P59.9 & Neonatal jaundice, unspecified & 3,161 & 364,750 & 168,853 & 104,532 & 747,173 & 117,029 & 557,682 & $2,063,180$ & $4,029,531$ & $(1,966,351)$ \\
\hline 7 & A01.0 & Typhoid fever & 7,638 & 573,884 & 214,609 & 202,047 & $1,097,418$ & $2,196,813$ & 4,044 & $4,296,454$ & $2,605,426$ & $1,691,028$ \\
\hline 8 & D36.7 & $\begin{array}{l}\text { Benign neoplasm, other specified } \\
\text { sites }\end{array}$ & $2,135,207$ & $1,296,637$ & $\mathbf{9 7 , 5 3 5}$ & 259,168 & $1,519,151$ & $1,133,989$ & 6,277 & $6,447,963$ & $4,303,346$ & $2,144,616$ \\
\hline 9 & 002.0 & $\begin{array}{l}\text { Blighted ovum and nonhydatidiform } \\
\text { mole }\end{array}$ & 508,290 & 152,632 & $\mathbf{7 4 , 8 5 0}$ & 333,054 & $1,491,505$ & $1,483,388$ & 130,658 & $4,174,376$ & $1,898,351$ & $2,276,025$ \\
\hline 10 & 042.0 & $\begin{array}{l}\text { Premature rupture of membranes, } \\
\text { onset of labour within } 24 \text { hours }\end{array}$ & $2,462,735$ & 295,845 & 187,241 & 424,235 & $2,090,408$ & $1,482,343$ & 86,869 & $7,029,676$ & $5,248,360$ & $1,781,316$ \\
\hline 11 & D21.0 & $\begin{array}{l}\text { Benign neoplasm, connective and } \\
\text { other soft tissue of head, face and } \\
\text { neck }\end{array}$ & $2,086,335$ & 249,240 & 75,583 & 328,712 & $1,407,225$ & $1,413,859$ & - & $5,560,954$ & $4,210,442$ & $1,350,512$ \\
\hline 12 & D24 & Benign neoplasm of breast & $2,420,349$ & 229,966 & 85,548 & 445,833 & $1,467,715$ & $1,276,153$ & - & $5,925,564$ & $5,701,672$ & 223,893 \\
\hline 13 & I63.9 & Cerebral infarction, unspecified & 25,070 & 799,074 & 276,468 & $1,454,248$ & $1,485,830$ & $1,919,556$ & 264,350 & $6,224,596$ & $5,328,444$ & 896,152 \\
\hline 14 & K65.9 & Peritonitis, unspecified & $2,364,815$ & 406,266 & 184,911 & 215,372 & $1,860,984$ & $1,625,393$ & - & $6,657,742$ & $6,518,658$ & 139,083 \\
\hline 15 & E11.5 & $\begin{array}{l}\text { Non-insulin-dependent diabetes } \\
\text { mellitus with peripheral circulatory } \\
\text { complications }\end{array}$ & $2,329,083$ & 518,999 & 189,864 & 533,931 & $1,758,683$ & $1,669,951$ & 14,474 & $7,014,986$ & $6,550,378$ & 464,608 \\
\hline 16 & K35.8 & $\begin{array}{l}\text { Acute appendicitis, other and } \\
\text { unspecified }\end{array}$ & $2,675,933$ & 394,156 & 155,632 & 232,593 & $1,866,571$ & $1,425,013$ & - & $6,749,898$ & $3,272,947$ & $3,476,951$ \\
\hline 17 & K40.9 & $\begin{array}{l}\text { Unilateral or unspecified inguinal } \\
\text { hernia, without obstruction or } \\
\text { gangrene }\end{array}$ & $2,333,324$ & 342,494 & 108,496 & 251,831 & $1,716,572$ & $1,537,329$ & - & $6,290,046$ & $\mathbf{5 , 1 3 7 , 7 8 9}$ & $1,152,257$ \\
\hline 18 & E11.9 & \begin{tabular}{|l|} 
Non-insulin-dependent diabetes \\
mellitus without complications
\end{tabular} & 14,362 & 633,714 & 268,047 & 531,631 & $1,314,274$ & $1,316,838$ & 26,543 & $4,105,410$ & $4,482,758$ & $(377,349)$ \\
\hline 19 & A90 & Dengue fever [classical dengue] & 4,383 & 591,523 & 193,564 & 327,646 & 950,494 & 598,545 & 18,625 & $2,684,781$ & $1,885,773$ & 799,008 \\
\hline 20 & 063.0 & Prolonged first stage of labour & $2,663,036$ & 320,404 & 147,408 & 536,935 & $1,977,375$ & $1,406,841$ & 89,650 & $7,141,650$ & $4,894,536$ & $2,247,114$ \\
\hline
\end{tabular}


From the results of the overall analysis, it shows that the value factor of Ummi's regular hospital rates compared to Ina-CBG's National Health Insurance rates is the cause of a large gap that causes the recording of the burden of correction for the difference in National Health Insurance which in the end is treated as a reduction in hospital income. If an Accounting treatment approach is taken from making journal of the transaction, it can be explained as follows:

- Journal of income from National Health Insurance patients when billing or invoice is issued at the hospital's regular rate:

$\begin{array}{cc}\text { Dr. Accounts Receivable National Health Insurance } \mathrm{xxx} \\ \text { Dr. Discount (if any) } & \mathrm{xx} \\ \text { Cr. Income } & \mathrm{xxx}\end{array}$

- Journal of charges for corrections to the difference between the regular hospital rates and Ina-CBG's National Health Insurance rates:
Dr. Difference in National Health Insurance
$\mathrm{xxx}$
Cr. Accounts Receivable National Health Insurance
$\mathrm{XXX}$

This tariff difference forms a correction by debiting the National Health Insurance Difference as an expense or cost of goods sold and crediting National Health Insurance Accounts Receivable. This fare difference is recorded in the next month or period after the service period. Furthermore, the difference between National Health Insurance will be net-off with the total revenue or as a deduction from the income in its reporting or in this case the hospital's Profit and Loss Report, because by nature the transaction is hospital income. The solution that must be taken by Ummi's hospital management in order to overcome the problem of the high gap between Ina-CBG's rates where this rate is in the form of a package or prosective with regular hospital rates is the need to calculate or recalculate in detail the unit cost or cost of treatment of each. diagnosis or medical action covered by National Health Insurance based on the elements or components of the burden included in determining the exact and ideal hospital margins but not also detrimental to the hospital, then of course the recognition of hospital revenues for National Health Insurance patients is more informative close to the real National Health Insurance rate.

For adjustments to the National Health Insurance rates, it is determined by the management below the general hospital rate with the consideration that the contribution to National Health Insurance patient services is greater than the general patient. Adjustments to the National Health Insurance rates begin by calculating the unit cost of each action on the diagnosis in detail. After obtaining the unit cost for this action, the hospital management will determine and impose the amount or value of the tariff for National Health Insurance services, which is of course a selling price that is not detrimental to the hospital but will be below the general hospital rate for non-National Health Insurance patients.

In order to fulfill the principle of matching cost against revenue in the preparation of Ummi hospital financial reports, the use of the Accrual Basis must be applied consistently in the process of recording all transactions that occur in the hospital, the recognition of revenues and expenses recorded in the same period or matching one with the same. Others so that the published financial reports are more rational and systematic.

\section{CONCLUSIONS AND SUGGESTIONS}

\subsection{Conclusion}

1. In recording income for financial statements, the Bogor UMMI hospital has used the Accrual Basis method in accordance withstatement of Financial Accounting Standards 23 concerning Income, income is recognized when the billing or invoice for patients is issued. Recognition of UMMI Bogor hospital income consists of outpatient income and inpatient income for general patients and National Health Insurance patients where the contribution of income from National Health Insurance patients is greater than general patients.

2. Recognition of hospital income from National Health Insurance patients using the regular rate value of the current general hospital master rate, resulting in a difference with the INA-CBG's National Health Insurance rate where the value of the National Health Insurance difference greatly affects the value of hospital income.

3. The results of the analysis show that there is a correction to the income recorded in the National Health Insurance Difference account where the loading is different for the period from the revenue recognition, so it is not in accordance with the principle of matching cost against revenue.

4. From the results of the analysis, the implications of the feedback period for hospital claims to National Health Insurance cause medical service charges to be recorded on a cash basis or not in accordance with the recording period of their income which shows that they are not in accordance with the principle of matching cost against revenue.

\subsection{Suggestion}

1) With the two applicable rates, namely the regular hospital rate and INA-CBG's National Health Insurance, the hospital management should 
calculate the correct unit cost and cost of treatment for each action in its medical services so that they can find out whether the INA-CBG's package rates are already cover the costs incurred by the hospital.

2) With the contribution of income from National Health Insurance patients that is greater than the general patient's income, the hospital management should organize a separate accounting process to accommodate the implementation of the Matching Cost Against Revenue principle and get a comprehensive and accurate solution, so that the right recording treatment can be determined for the patient National Health Insurance.

3) In order for the Matching Principle to be applied in accounting records, the hospital management should make a policy to estimate corrections from National Health Insurance based on historical data and recorded in the same month as the recording of income, so that the Accrual Basis recording method can be applied consistently in accordance with the Accounting Standards.

4) Medical service expenses which include the services of doctors, nurses and other medical teams whose records are not in accordance with the matching principle due to the National Health Insurance feedback period so that it is accrued based on historical data by referring to policies from hospital management. 4. The same research can be carried out at other hospitals that serve National Health Insurance patients with a larger income contribution than general patients so that the problem of the gap between regular hospital rates and INA-CBG's National Health Insurance rates can get a comprehensive and accurate solution so that the recording treatment can be determined.

\section{REFERENCE}

1. Hongren, Charles $\mathrm{T}$, Harrison, Walter $\mathrm{T}$. Accounting. First Volume, Seventh Edition. Jakarta: Erlangga Publisher; 2007.

2. Kieso DE, Weygandt JJ, Warfield TD. Intermediate Accounting. Jakarta: Erlangga; 2016.

3. Beechy TH. Does full accrual accounting enhance accountability? The Innovation Journal: The Public Sector Innovation Journal. 2007;12(3):1-8.

4. Mulyadi. Accounting System. Jakarta: Four Salemba. Soemarso. Accounting an Introduction. Jakarta: Four Salemba; 2008.

5. Indonesian Accountants Association. Indonesian Accounting Standards. Jakarta: Four Salemba; 2015.

6. Belkaoui, Ahmed. Accounting Theory. Second Edition First Volume. Translation of Herman Wibowo and Marianus Sinaga. Jakarta: Erlangga; 2004. 